\title{
OPTIMISATION DE PLANS DE FINANCEMENT IMMOBILIERS *
}

\author{
FRÉDÉRIC GARDI ${ }^{1}$
}

Résumé. La finance de marché est devenue un des domaines d'application privilégiés de la recherche opérationnelle. D'un autre côté, rares sont les applications touchant la banque de détail, tournée vers le grand public. Dans ce papier, nous abordons un problème d'actualité dans le secteur bancaire français : l'optimisation de plans de financement immobiliers. Le travail que nous présentons a été effectué dans le cadre du développement par la société Experian-Prologia d'une nouvelle application d'instruction de prêts immobiliers pour une grande banque française. Notre module d'optimisation, au cœur de cette application, est aujourd'hui déployé dans les 2200 agences de cette banque et permet la simulation et le montage de plusieurs milliers de plans de financement immobiliers chaque mois.

Mots Clés. banque/finance, crédit/prêt immobilier, mathématiques actuarielles, programmation linéaire mixte, application/logiciel .

\begin{abstract}
Market finance has become a major application field of operations research. On the other hand, rare are the applications impacting the retail banking, turned toward general public. In this paper, a topical problem in the French banking sector is approached : the optimization of real-estate financial plans. The present work was done in the context of the development by the firm Experian-Prologia of a new mortgages processing application for an important French bank. Our optimization module, in the heart of this application, is now deployed in the 2200 branches of this bank and enables to simulate and assembly several thousands of real-estate financial plans every month.
\end{abstract}

Keywords. Banque/finance, crédit/mortgage, actuarial mathematics, linear programmation, application/software.

Classification Mathématique. 90B90, 91B28, 90C90.

Received October 14, 2007. Accepted May 05, 2010.

* Les travaux de recherche et développement exposés dans ce papier ont été réalisés alors que l'auteur travaillait au sein de la société Experian-Prologia SAS, Marseille, France.

1 Bouygues e-lab, 32 avenue Hoche, 75008 Paris, France; fgardi@bouygues.com 


\section{INTRODUCTION}

Le financement d'une opération immobilière requiert d'importantes sommes d'argent, généralement prêtées par une banque ou un organisme de crédit. Si certaines banques peuvent proposer des financements adossés à des assemblages de prêts, ceux-ci demeurent limités tant par la technicité de ces prêts que par les outils de calcul classiques qui aident à les composer. Dans leur grande majorité, les plans de financement se contentent d'un seul prêt dont l'échéance est constante sur la durée de celui-ci. Une grande banque française, qui avait préalablement fait évoluer sa gamme de produits immobiliers vers des technicités permettant plus de souplesse dans le remboursement, souhaitait pratiquer des assemblages de prêts afin de proposer des plans de financement compétitifs en ces temps de flambée des prix de l'immobilier. Pour aider les chargés de clientèle dans ce travail et être certain de proposer les meilleures solutions à ses clients, cette banque a décidé d'intégrer à son nouveau système informatique d'instruction de prêts immobiliers - entièrement développé par la société Experian-Prologia, spécialiste de l'aide à la décision pour le secteur bancaire - un module d'optimisation de plans de financement immobiliers. Ce module, basé sur une approche par programmation linéaire mixte, a demandé près de deux ans de recherche et développement et est progressivement entré en exploitation dans le réseau des 2200 agences de cette banque début 2006. Depuis, plus de 10000 chargés de clientèle ont été formés à l'utilisation de ce logiciel informatique, qui permet aujourd'hui de construire de façon automatique et optimisée plusieurs milliers de plans de financement chaque mois.

À notre connaissance, aucune étude n'a encore été publiée sur ce sujet dans la littérature scientifique ou spécialisée, hormis une note sommaire traitant d'un problème apparenté [1]. Le présent papier, dont des versions préliminaires apparaissent dans des actes de colloques $[6,7]$ ainsi que dans la revue française d'actuariat [8], fait la synthèse complète des travaux que nous avons menés sur le sujet. Avant de définir ce que nous entendons par optimisation de plans de financement immobiliers, il nous faut préciser ce qu'est un prêt, ainsi que les différents objets qui s'y rattachent, comme les assurances ou les garanties. À ce propos, nous recommandons au lecteur intéressé le lexique du financement de l'immobilier de Fortunat et al. [5].

\subsection{LES PRÊTS IMMOBILIERS}

La forme d'emprunt la plus classique pour financer une opération immobilière (acquisition d'un bien immobilier, construction, travaux) est le crédit amortissable, qui donne lieu à un remboursement du capital prêté par des échéances périodiques, dont le montant est composé d'une fraction du capital emprunté et d'intérêts calculés sur le capital restant dû au titre de la période. Nous ne parlerons pas ici des prêts relais ou encore des prêts in fine qui, contrairement aux prêts amortissables, voient le montant de leurs échéances réduit au seul paiement des intérêts (voire d'une partie seulement), le remboursement de la dette se faisant en une seule fois 


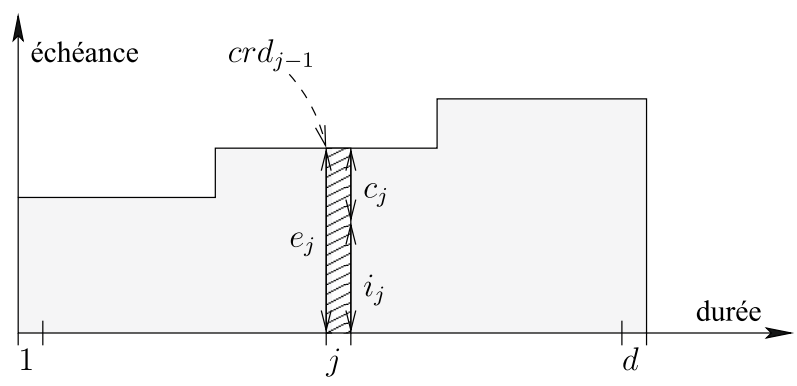

Figure 1. Représentation graphique d'un prêt.

à la fin du prêt. Le prêt relais sert à financer une opération immobilière dans l'attente de la vente d'un bien devant permettre le remboursement de tout ou partie du capital emprunté (par exemple en cas de déménagement); le prêt in fine, généralement adossé à un produit d'épargne par capitalisation, est destiné à financer un placement immobilier de type investissement locatif et n'a donc de sens que dans le contexte d'une optimisation fiscale, qui n'est pas notre propos ici.

De manière générale, un prêt amortissable a un montant $m$ (aussi appelé capital initial), une durée $d$ et des échéances périodiques de remboursement $e_{1}, \ldots, e_{d}$. Le montant de chaque échéance $e_{j}$ est composé d'une part $c_{j}$ de capital amorti à l'issue de la période $j$ et d'intérêts $i_{j}$ calculés sur le capital restant dû au titre de cette même période (voir Fig. 1). Ainsi, le calcul des intérêts est basé sur la récurrence

$$
\begin{aligned}
& i_{1}=T_{1} \cdot m \\
& i_{j}=T_{j} \cdot\left(m-\sum_{k=1}^{j-1} c_{k}\right) \quad \forall j=2, \ldots, d
\end{aligned}
$$

où $T_{j}$ représente le taux d'intérêt du prêt à la période $j$ et $c r d_{j-1}=m-\sum_{k=1}^{j-1} c_{k}$ le capital restant dû à l'issue de la période précédente. La condition $c r d_{d}=0$, soit $m=\sum_{j=1}^{d} c_{j}$, garantit alors que la totalité du capital emprunté est amorti après le paiement de la dernière échéance $e_{d}$. Autrement dit, l'amortissement d'un prêt suit les équations

$$
\begin{aligned}
& c_{1}=e_{1}-T_{1} \cdot m \\
& c_{j}=e_{j}-T_{j} \cdot\left(m-\sum_{k=1}^{j-1} c_{k}\right) \quad \forall j=2, \ldots, d
\end{aligned}
$$


auxquelles il faut ajouter les contraintes

$$
\begin{aligned}
& c_{1}, \ldots, c_{d} \geq 0 \\
& m=\sum_{j=1}^{d} c_{j}
\end{aligned}
$$

afin d'assurer sa validité (remboursement périodique des intérêts) ainsi que sa terminaison (capital restant dû nul à l'issue de la période $d$ ). La périodicité naturelle de remboursement pour des particuliers est le mois, mais d'autres périodicités peuvent être envisagées comme le trimestre, le semestre ou l'année.

Les prêts immobiliers amortissables peuvent être classés en deux catégories. Les premiers sont les prêts du secteur libre, non réglementés, qui sont consentis par les établissements de crédit sous différentes formes pour répondre aux besoins de la clientèle et aux contraintes du marché. Les seconds, les prêts aidés et réglementés par l'État français, sont soumis à une réglementation stricte, offrant en contrepartie de nombreux avantages aux emprunteurs qui peuvent y prétendre. Les prêts aidés par l'État possèdent en général des caractéristiques plus complexes que les prêts du secteur libre, dues aux réglementations spécifiques auxquelles ils sont soumis. De nombreux types de prêts réglementés existent. Dans le cas présent, nous avons eu à traiter les six suivants : le prêt épargne logement construit à base de plans épargne logement (PEL) ou à base de comptes épargne logement (CEL), le prêt à $0 \%$ Ministère du logement (PTZ), le récent prêt Paris logement $0 \%$ (PPL) subventionné par la ville de Paris, les prêts conventionnés (PC) ou à l'accession sociale (PAS). Chacun de ces types de prêts est soumis à des contraintes particulières, souvent complexes, en sus des contraintes d'amortissement décrites ci-dessus. Nous détaillerons par la suite les contraintes auxquelles sont soumis les différents prêts que nous avons rencontrés, du secteur libre ou réglementé.

\subsection{Assurances, garanties et autres frais}

Aux échéances versées pour rembourser l'emprunt peut éventuellement s'additionner une ou plusieurs primes d'assurance couvrant divers risques (décès, invalidité, incapacité, perte d'emploi). La plupart des assurances sont calculées sur capital initial (CI), bien qu'apparaissent aujourd'hui des assurances sur capital restant dû (CRD). Dans le cas d'une assurance sur CI de taux périodique $A_{j}$, les équations (1.1) et (1.2) d'amortissement deviennent :

$$
\begin{aligned}
& c_{1}=e_{1}-T_{1} \cdot m-A_{1} \cdot m \\
& c_{j}=e_{j}-T_{j} \cdot\left(m-\sum_{k=1}^{j-1} c_{k}\right)-A_{j} \cdot m \quad \forall j=2, \ldots, d .
\end{aligned}
$$


Dans le cas d'une assurance sur CRD, le taux $A_{j}$ de l'assurance s'additionne simplement au taux du prêt sur la période :

$$
\begin{aligned}
& c_{1}=e_{1}-\left(T_{1}+A_{1}\right) \cdot m \\
& c_{j}=e_{j}-\left(T_{j}+A_{j}\right) \cdot\left(m-\sum_{k=1}^{j-1} c_{k}\right) \quad \forall j=2, \ldots, d .
\end{aligned}
$$

Une garantie est un moyen juridique dont le but est d'assurer au créancier une plus grande sécurité quant au remboursement de sa créance. Généralement exigée dans le cadre d'un prêt immobilier, la prise d'une garantie réelle a un coût qui, tout comme celui des assurances, n'est pas négligeable; d'autant qu'il s'avère parfois nécessaire de financer celui-ci au travers de l'emprunt lui-même (lorsque l'apport personnel de l'emprunteur est faible). En définitive, le coût d'une garantie peut s'exprimer comme une fonction linéaire par morceaux du montant qu'elle couvre. De nombreux autres frais viennent s'ajouter au coût total de l'opération. Les seuls frais qui nous importent ici sont les frais relatifs au montage du plan de financement : les frais de dossier. Liés au coût de l'édition de l'offre de chaque prêt composant le plan de financement, leur montant est égal à un pourcentage du montant du prêt, borné par un minimum et un maximum.

La modélisation et le traitement des assurances, garanties et frais au sein du problème d'optimisation de plans de financement immobiliers fera l'objet d'une section spécifique.

\subsection{Optimisation de Plans De financement}

De façon informelle, construire un plan de financement consiste à assembler un ensemble de prêts, en déterminant leurs montants, durées et échéances, ainsi que les assurances et garanties qui s'y rattachent, de façon à satisfaire un besoin de financement. Afin d'être compétitif, il est demandé aux chargés de clientèle de rechercher la solution de financement la plus adaptée aux besoins de leurs clients et la moins chère possible, par rapport aux prêts, assurances et garanties qui peuvent être mis à sa disposition par la banque. De là, il y a deux façons de définir ce que l'on entend par optimiser le plan de financement.

La première est la suivante : le client a une capacité maximale de remboursement (qui peut être variable dans le temps) et l'on souhaite construire un plan de financement de coût minimum (c'est-à-dire qui minimise la somme des intérêts versés et des coûts additionnels engendrés par les assurances, garanties ou autres frais) dont les échéances sont inférieures à cette capacité. Nous insistons sur le fait que les échéances de remboursement souhaitées par le client peuvent varier d'une période à l'autre, le but étant de proposer à celui-ci une solution adaptée à l'évolution dans le temps de sa capacité de remboursement (au moins dans un futur proche). Ces variations peuvent également être le fait de diverses charges (prêts immobiliers déjà contractés, crédits à la consommation). 
La seconde peut être formulée comme suit : le client souhaite que son remboursement s'étale sur une certaine durée. Le but est alors de trouver un plan de financement qui, ne dépassant pas cette durée, minimise l'échéance la plus haute du plan. Nous cherchons en fait à lisser les échéances payées par le client sur la durée du plan de financement. Ce mode d'optimisation, qui est le complémentaire du précédent, permet en outre de déterminer la capacité minimale de remboursement nécessaire au client pour amortir son emprunt sur la durée souhaitée.

De plus, la possibilité doit être offerte au chargé de clientèle de poser lui-même certaines contraintes sur le plan de financement ou certains produits le composant (prêts, assurances, garanties). Par exemple, celui-ci peut contraindre la durée ou le montant de certains prêts, voire forcer leur inclusion au sein de la solution de financement; cela permet notamment de proposer au client des plans mêlant prêts à taux variables, moins chers à court terme, et prêts à taux fixes, plus sûrs à long terme.

En définitive, on peut observer l'équivalence des deux problèmes d'optimisation en terme de difficulté : optimiser le coût d'un plan de financement contraint par une capacité de remboursement donnée n'est pas moins difficile que lisser un plan de financement sur une durée souhaitée et vice versa. Dans les deux cas, une dimension du problème est fixée (l'échéance ou la durée) et l'autre est une variable de l'optimisation. Ainsi, dans son essence, le problème de décision peut être formulé comme suit. Soit un besoin de financement $B$, une capacité de remboursement $E_{j}$ chaque mois $j$, une durée maximale de remboursement $D$ et un ensemble de $n$ prêts $P_{1}, \ldots, P_{n}$. La question est : existe-t-il un plan de financement $\mathcal{P}$ ? C'est-à-dire un sous-ensemble $\mathcal{P}$ de ces $n$ prêts tel que : (i) la somme des montants des prêts du plan $\mathcal{P}$ couvre le besoin de financement $B$, (ii) la somme des mensualités des prêts du plan n'excède pas la capacité $E_{j}$ chaque mois $j$, (iii) la durée de chaque prêt du plan ne dépasse pas la durée maximale $D$, (iv) chaque prêt du plan respecte les contraintes qui lui sont propres (en particulier les équations d'amortissement (1.1)(1.4)). Les contraintes (i), (ii), (iii) sont appelées "contraintes de plan", tandis que les contraintes (iv) sont appelées "contraintes de prêt". La mensualité du plan un mois donné correspond à la somme des mensualité des prêts composant le plan ce même mois; la durée du plan correspond à la durée du prêt le plus long du plan de financement.

\section{RÉSOlution : Difficultés ET IDÉES}

La difficulté du problème résulte de plusieurs facteurs. La modélisation d'un prêt fait apparaître des variables discrètes, les durées, qui introduisent une combinatoire dans le problème (d'autant que la périodicité considérée ici est mensuelle). Nous verrons que cet aspect combinatoire est source de la difficulté intrinsèque du problème d'optimisation de plan de financement, dans le cas général. À cette difficulté de nature combinatoire s'ajoute le fait que plusieurs contraintes réglementaires ou métier ont un caractère non linéaire, ou parfois même sont difficiles à exprimer sous une forme numérique ou logique. 
À cela s'ajoutent trois difficultés opérationnelles. La première est l'aspect temps réel de la résolution. En effet, le chargé de clientèle doit pouvoir présenter dans l'immédiat une ou plusieurs solutions au client, ce qui ne laisse qu'un temps de calcul très court au module d'optimisation (moins d'une seconde). Ensuite, robustesse et stabilité de l'algorithme sont exigées : les plans de financement optimisés sont voués à être commercialisés en l'état, sans retouche de la part du chargé de clientèle. Nous insistons sur le fait que, contrairement aux problèmes généralement rencontrés dans le domaine financier où l'on résonne sur des masses monétaires, les montants ne peuvent pas ici être explicitement considérés comme des variables rationnelles pour des raisons à la fois réglementaires et commerciales : il est impératif que le client puisse connaître la composition exacte des échéances qu'il paye (en particulier la part des intérêts versés et la part de capital amorti), et ce au centime près. Pour cela, la banque a défini des règles d'arrondi précises, afin d'assurer la compatibilité des plans de financement tout au long de la chaîne de gestion. Enfin, une certaine généricité est souhaitée dans l'approche du problème (modélisation et résolution) de façon à pouvoir intégrer rapidement de nouveaux produits ou de nouvelles contraintes réglementaires, limitant ainsi des approches heuristiques dédiées.

Les actuaires de la banque pour laquelle nous avons travaillé ont développé il y a quelques années un outil qui permet l'assemblage d'un nombre limité de prêts possédant des caractéristiques précises, en procédant à une énumération orientée métier des solutions. Ici, la définition générique du problème couplée au cadre opérationnel très strict empêche toute tentative de résolution en temps réel par force brute. L'idée maîtresse sur laquelle repose notre schéma de résolution est la modélisation du problème sous la forme d'un programme linéaire mixte à la combinatoire réduite (par filtrage de domaines et adjonction de coupes) permettant une résolution directe par un algorithme classique de séparation et évaluation. L'heuristique complète se décompose alors en trois phases : une première phase effectue certains traitements préliminaires (calculs de bornes), la deuxième détermine le profil d'un plan de financement optimal (par programmation linéaire mixte) et enfin la troisième calcule un plan commercialisable s'appuyant sur ce profil (à l'aide de règles métier). Cette approche permet une résolution quasi exacte du problème en temps réel, le nombre de prêts susceptibles d'intégrer un plan de financement ne dépassant pas la dizaine en pratique. Notons qu'en pratique, le programme linéaire mixte est résolu à l'aide de la librairie libre GLPK [11], tant pour des raisons économiques (coût d'exploitation) que pratiques (code source disponible facilitant intégration et maintenance).

La deuxième phase, au centre du processus de résolution, consiste à calculer un plan de financement non admissible, mais très proche d'un plan optimal en terme de coût. Si aucun profil n'est trouvé, l'algorithme stoppe et retourne une liste de messages permettant d'expliquer cet échec (c'est-à-dire quelles sont les contraintes susceptibles d'être à l'origine de l'absence de solution); si un profil est déterminé, alors la dernière phase est chargée de retoucher le plan de financement obtenu afin de le rendre viable d'un point de vue opérationnel et commercial (c'est-à-dire respecter les règles d'arrondi, ainsi que diverses règles esthétiques). Ce dernier point 


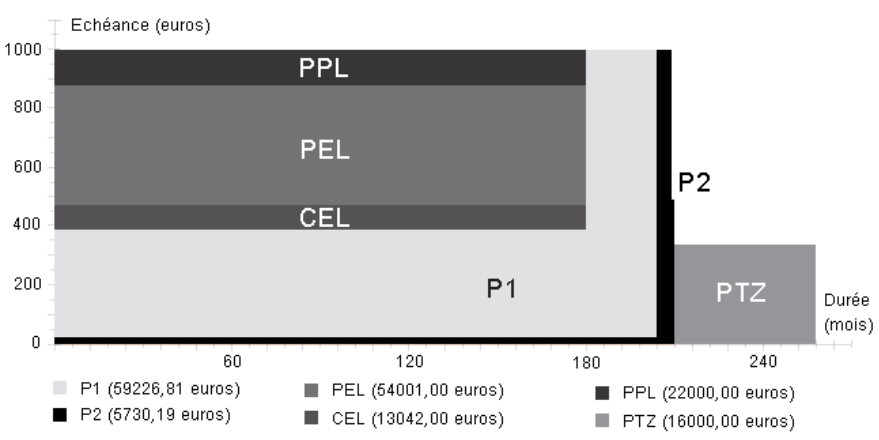

Figure 2. Un plan de financement optimisé à base de 6 prêts : deux prêts du secteur libre (P1, P2), deux prêts épargne logement (PEL, CEL) et deux prêts à taux zéro (PPL, PTZ).

est dû au fait que les solutions retournées par le module d'optimisation peuvent être difficiles à appréhender pour les chargés de clientèle, qui n'ont généralement qu'une formation scientifique de base, et donc à expliquer à leurs clients. La Figure 2 montre un exemple de plan de financement complexe obtenu en temps réel par le module d'optimisation, couvrant un besoin de financement de 170000 euros pour une capacité mensuelle de remboursement de 1000 euros.

La conception d'un algorithme sujet à de tels impératifs de performance requiert un important travail d'ingénierie algorithmique et logicielle, que nous ne pouvons entièrement détailler ici. Par conséquent, nous nous focaliserons sur le modèle linéaire mixte au cœur de l'heuristique et les propriétés employées pour le réduire de façon à le rendre soluble en pratique. Un résultat fondamental est notamment démontrée caractérisant les plans de financement optimaux construits à base de prêts du secteur libre (Prop. 3.10). Le modèle linéaire mixte est semblable que l'on cherche à minimiser le coût du plan $\mathcal{P}$ ou bien à lisser celui-ci sur la durée $D$, seuls les contraintes et objectifs du plan sont modifiées. Ainsi, la description du modèle s'étale sur les quatre sections suivantes : la Section 3 portant sur les propriétés et la modélisation des prêts du secteur libre, la Section 4 décrivant la modélisation des prêts réglementés, la Section 5 portant sur le traitement des assurances, des garanties et des frais, la Section 6 décrivant la modélisation des contraintes et objectifs du plan. Nous conclurons le papier par la présentation de quelques cas pratiques et résultats numériques démontrant le caractère effectif de notre approche, ainsi que par une ouverture vers des problématiques connexes de financement, susceptibles d'être abordées d'une manière similaire.

\section{LeS PRÊTS DU SECTEUR LIBRE}

Les prêts du secteur libre possèdent de façon générale les caractéristiques suivantes : un montant minimum $M_{\min }$, un montant maximum $M_{\max }$, une durée minimum $D_{\min }$, une durée maximum $D_{\max }$, un montant d'amortissement mensuel 
minimum $C_{\min }$. En pratique, le montant minimum est bas (de l'ordre du millier d'euros) et le montant maximum est supposé infini pour les prêts standards (ceux pour lesquels sont pratiqués les taux du marché); le montant d'amortissement mensuel minimum est symbolique, puisque étant d'un euro seulement.

Une caractéristique importante du prêt dans le secteur libre est sa grille de taux. À chaque prêt est associée une grille qui, pour chaque durée $D_{\min } \leq d \leq D_{\max }$, donne le taux $T_{d}$ d'emprunt sur cette durée. Une propriété intéressante est que les grilles de taux à moyen/long terme sont croissantes : pour tout $d^{\prime}>d$, nous avons $T_{d^{\prime}} \geq T_{d}$. En effet, plus la durée de l'emprunt est longue, plus le risque est grand pour la banque, plus le taux consenti est élevé. En pratique, la banque définit les taux de ses prêts pour les 8 plages de durées suivantes, exprimées en années : 7, 10, $12,15,17,20,25,30$. Nous noterons $G$ le nombre de plages de durées des grilles de taux.

Une autre caractéristique fondamentale d'un prêt est le profil de ses échéances (montant et durée de chaque palier, pourcentage de variation entre les montants des paliers, etc.). Deux types naturels de profil sont considérés ici : constant ou libre. Les échéances d'un prêt à profil constant doivent toutes être égales sur la durée de celui-ci, tandis que celles d'un prêt à profil libre peuvent varier librement au cours de sa durée. Aujourd'hui encore de nombreuses banques ne composent qu'avec des prêts à profil constant, bien souvent car leur système d'information (et plus particulièrement leurs outils de calcul et de simulation de plan de financement) ne permettent pas de gérer les prêts à paliers. Du fait de leur structure rigide, les prêts à profil constant ne permettent pas d'assemblages sur mesures, comme le souhaitent beaucoup de clients aujourd'hui, ni de véritable optimisation. Pour cette raison, la banque les a progressivement remplacés par des prêts à profil libre.

Dans cette section, nous aborderons chacun des deux types de prêts séparément, mais selon le même mode opératoire. Après avoir dégagé certaines propriétés structurelles et analysé la complexité du problème, nous décrivons comment les modéliser à l'aide de contraintes linéaires mixtes dans un cadre opérationnel.

\subsection{LES PRÊTS À PROFIL CONSTANT}

Les prêts à profil constant étaient jusqu'à présent la norme dans le monde du crédit immobilier. Ces prêts se caractérisent par un montant $m$, une durée $d$, une échéance $e$ constante sur cette durée et un taux $T_{d}$ (supposé fixe sur la durée du prêt). Une formule analytique reliant ces quatre quantités peut alors être déduite des équations d'amortissement (1.1)-(1.4). Cette élégante formule, bien connue des actuaires et présente dans tous les livres de mathématiques financières $[4,10]$, est la suivante :

$$
m=e \cdot \sum_{j=1}^{d} \frac{1}{\left(1+T_{d}\right)^{j}}=e \cdot \frac{\left(1+T_{d}\right)^{d}-1}{T_{d}\left(1+T_{d}\right)^{d}}=e \cdot \operatorname{VAN}\left(1, d, T_{d}\right)
$$

où $\operatorname{VAN}(d, f, t)=\sum_{j=d}^{f} \frac{1}{(1+t)^{j}}$, appelé valeur actuelle nette, peut être interprété comme la valeur actuelle d'un euro versé mensuellement du mois $d$ au mois $f$ au 
taux $t$ (se reporter à [4,10] pour plus de détails). Cette formule sera abondamment employée pour modéliser nos différents prêts. En effet, une remarque clé est qu'à durée fixée, le taux l'étant lui aussi, l'équation (3.9) devient linéaire en le montant et l'échéance.

\subsubsection{Propriétés et complexité}

Un inconvénient des prêts à profil constant est qu'ils ne peuvent généralement pas saturer la capacité de remboursement, en particulier lorsque celle-ci est variable dans le temps. Admettons que le client ait un besoin de financement $B$ et une capacité de remboursement donnée sous la forme de $k$ paliers $\left(D_{j}, E_{j}\right)$. Pour $j=1, \ldots, k$, posons $\bar{E}_{j}=\min \left\{E_{j}, \bar{E}_{j-1}\right\}$. Immédiatement, nous pouvons faire l'observation suivante.

Observation 3.1. Tout plan de financement composé seulement de prêts à profil constant ne peut saturer qu'une capacité $\bar{E}_{j}$ pour tout palier $j=1, \ldots, k$. Comme $\bar{E}_{j} \geq \bar{E}_{j+1}$ pour tout $j=1, \ldots, k-1$, la capacité pouvant être saturée par un ensemble de prêts à profil constant induit un escalier descendant. En d'autres termes, toute augmentation future de la capacité de remboursement du client n'est en rien profitable à celui-ci.

En outre, nous montrons que déterminer l'existence d'un plan de financement est un problème difficile lorsque seuls des prêts à profil constant sont susceptibles d'intégrer celui-ci. Soit $n$ prêts $P_{1}, \ldots, P_{n}$ à profil constant, un besoin de financement $B$ et une capacité de remboursement $E_{j}$ chaque mois $j$. La question est : existe-t-il un plan de financement $\mathcal{P}$ qui peut satisfaire d'une part les contraintes de prêt, soit pour tout $P_{i} \in \mathcal{P}$ :

$$
\begin{aligned}
& M_{\min _{i}} \leq m_{i} \leq M_{\max _{i}} \\
& D_{\min _{i}} \leq d_{i} \leq D_{\max _{i}} \\
& m_{i}=e_{i} \cdot \operatorname{VAN}\left(1, d_{i}, T_{i}\right)
\end{aligned}
$$

et d'autre part les contraintes de plan, soit $\sum_{P_{i} \in \mathcal{P}} m_{i} \geq B$ et $\sum_{P_{i} \in \mathcal{P}} e_{i} \leq E_{j}$ pour chaque mois $j$.

Proposition 3.2. Déterminer un plan de financement à base de prêts à profil constant est un problème $\mathcal{N} \mathcal{P}$-complet, même si la capacité $E_{j}$ est identique chaque mois $j$ et que $M_{\min _{i}}=M_{\max _{i}}, D_{\min _{i}}=D_{\max _{i}}$ et $T_{i}=0$ pour tout prêt $P_{i}$.

Démonstration. La réduction se fait depuis le problème $\mathcal{N} \mathcal{P}$-complet du sac-àdos [9]. Soit un ensemble de $n$ objets, chacun ayant un prix $p_{i}$ et une taille $t_{i}$ entiers. Le problème du sac-à-dos consiste à déterminer un sous-ensemble de ces $n$ objets de sorte que la somme de leurs prix soit supérieure à un entier $P$, mais que la somme de leurs tailles reste inférieure à un entier $T$. Ce problème reste $\mathcal{N} \mathcal{P}$-complet si l'on considère que $p_{i}$ est un multiple de $t_{i}$ pour chaque objet.

Pour chaque objet, définissons un prêt $P_{i}$ tel que $M_{\min _{i}}=M_{\max _{i}}=p_{i}, D_{\min _{i}}=$ $D_{\max _{i}}=p_{i} / t_{i}$ et $T_{i}=0$. Le taux étant nul, l'échéance du prêt $P_{i}$ est égale à son montant divisé par sa durée, soit ici $t_{i}$. Ensuite, posons le besoin $B$ de financement comme étant égal à $P$ et la capacité $E_{j}$ de remboursement comme étant égale à 


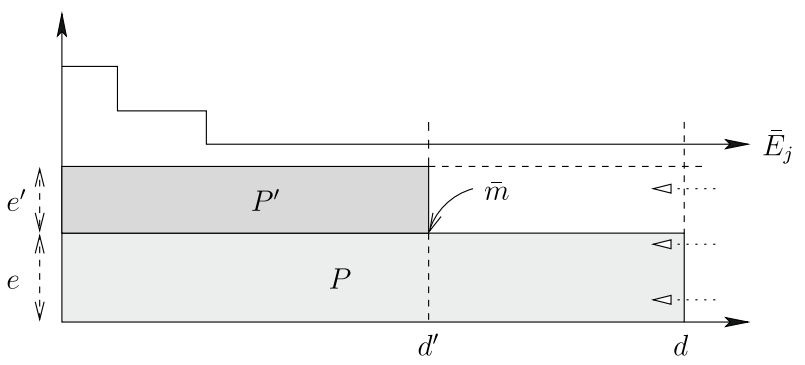

Figure 3 . Les prêts $P$ et $P^{\prime}$ à profil constant avec $t^{\prime}>t$.

$T$ pour tout $j$. Il est alors aisé de vérifier qu'il existe un plan de financement si et seulement si le problème du sac-à-dos a une solution.

Comme nous l'avons évoqué précédemment, les montants minimums (resp. maximums) des prêts peuvent être supposés nuls (resp. infinis) en pratique. Bien que nous ne soyons pas parvenus à élucider la complexité du problème dans ce cas, c'est-à-dire sans contrainte de montant sur les prêts, nous avons pu établir les propriétés suivantes. Dans les deux lemmes suivants, les prêts $P$ et $P^{\prime}$ sont considérés comme à profil constant et sans contrainte de montant.

Lemme 3.3 (dominance). Si un prêt $P$ à taux $t$ appartient à un plan de financement optimal et a comme date de fin d, alors ce dernier ne peut contenir un prêt $P^{\prime}$ de taux $t^{\prime}>t$ ayant comme date de fin $d^{\prime} \leq d$, dès lors que la capacité de remboursement $\bar{E}_{j}$ en $d^{\prime}$ est la même qu'en $d$.

Démonstration. Supposons que le prêt $P\left(\operatorname{resp} . P^{\prime}\right)$ ait un montant $m$ (resp. $\left.m^{\prime}\right)$ et une échéance $e$ (resp. $e^{\prime}$ ) et notons $\bar{m}$ le capital restant dû à l'issue du mois $d^{\prime}$ sur le prêt $P$ (voir Fig. 3). Nous affirmons qu'un plan de coût moindre peut être obtenu en empruntant le montant $m+m^{\prime}$ sur le prêt $P$ et en remboursant à échéance constante $e+e^{\prime}$. Tout d'abord, le capital restant dû à l'issue du mois $d^{\prime}$ dans ce cas est strictement inférieur à $\bar{m}$ (puisque le capital $m^{\prime}$ est maintenant amorti au taux $t<t^{\prime}$ ). Ensuite, ce capital restant dû est amorti par des mensualités constantes d'un montant $e+e^{\prime} \leq \bar{E}_{j}$ (et non plus d'un montant $e$ ), ce qui permet d'abaisser la durée du prêt $P$ et donc le coût du plan (les grilles étant croissantes, diminuer la durée du prêt ne peut être que profitable).

Ce lemme, qui s'apparente à une règle de dominance, ne tient plus lorsque la capacité de remboursement en $d^{\prime}$ est strictement supérieure à la capacité $\bar{E}_{j}$ en $d$, car il est possible que l'échéance $e^{\prime}$ soit telle que $e+e^{\prime}>\bar{E}_{j}$.

Lemme 3.4. Si un prêt $P$ à taux $t$ appartient à un plan de financement optimal et a comme durée $d$, alors tout prêt $P^{\prime}$ de taux $t^{\prime}<t$ et de durée $d^{\prime} \leq d$ est tel que $d^{\prime}$ est une date de variation de taux dans la grille de $P^{\prime}$ ou une date de variation de capacité de remboursement $\bar{E}_{j}$. 
Démonstration. Admettons que la durée $d^{\prime}$ soit inférieure d'au moins un mois à la plus petite de ces deux dates. Un plan de moindre coût peut être obtenu en transférant une fraction du capital emprunté du prêt $P$ vers le prêt $P^{\prime}$, puisque $t^{\prime}<t$ (et ce même si la durée du prêt $P$ est diminuée, puisque les grilles sont croissantes). L'augmentation du montant du prêt $P^{\prime}$ peut se faire à échéance constante, en allongeant sa durée d'un mois.

Des deux lemmes précédents, nous tirons la proposition suivante.

Proposition 3.5. Tout plan de financement optimal composé seulement de prêts à profil constant sans contrainte de montant satisfait les deux assertions suivantes lorsque la capacité de remboursement $E_{j}$ est identique chaque mois $j$ :

(a) l'ordre induit par les taux des prêts du plan est le même que l'ordre induit par les durées des prêts;

(b) hormis la durée du prêt le plus long, les durées des prêts coïncident avec des dates de variation de taux dans la grille.

\subsubsection{Modélisation par contraintes linéaires mixtes}

Dans cette section, nous décrivons comment sont modélisés les prêts à profil constant au sein du programme linéaire mixte. À l'état brut, le modèle est le suivant. À chaque prêt $P$ à profil constant est associée une variable booléenne $p \in\{0,1\}$ de manière à ce que $p=1$ si le prêt est inclus dans le plan de financement, et $p=0$ dans le cas contraire. La durée du prêt est alors "éclatée" en autant de variables booléennes $d_{D_{\min }}, \ldots, d_{D_{\max }} \in\{0,1\}$ que ce qu'il y a de mois entre $D_{\min }$ et $D_{\max }$ : la variable $d_{j}$ prendra la valeur 1 si le prêt $P$ a une durée de $j$ mois, et la valeur 0 sinon. Afin qu'une et une seule variable $d_{j}$ ne soit égale à 1 , et ce lorsque le prêt en question appartient à la solution de financement, nous posons la contrainte

$$
d_{D_{\min }}+\cdots+d_{D_{\max }}=p
$$

À chaque variable $d_{j}$ sont ensuite associées les variables rationnelles $m_{j}$ et $e_{j}$ correspondant respectivement au montant emprunté sur le prêt et à l'échéance mensuelle permettant le remboursement sur la durée $j$. Pour tout $j=D_{\min }, \ldots, D_{\max }$, nous posons alors les contraintes

$$
\begin{aligned}
& d_{j} \cdot M_{\min } \leq m_{j} \leq d_{j} \cdot M_{\max } \\
& m_{j}=e_{j} \cdot \operatorname{VAN}\left(1, j, T_{j}\right) \\
& e_{j} \geq T_{j} \cdot m_{j}+C_{\min } \cdot d_{j} .
\end{aligned}
$$

Notons que ces deux dernières contraintes sont bien linéaires puisque $M_{\min }, M_{\max }$ et $\operatorname{VAN}\left(1, j, T_{j}\right)$ sont des termes constants. En outre, le lecteur peut vérifier que lorsque la variable $d_{j}$ associée à la durée $j$ est active $\left(d_{j}=1\right)$, toutes les contraintes associées aux autres durées sont inactives $\left(m_{j^{\prime}}=0\right.$ et $e_{j^{\prime}}=0$ pour tout $j^{\prime} \neq j$, les $e_{j^{\prime}}$ étant minimisés). La contrainte (3.12) décrit l'amortissement du prêt sur la durée $j$ au taux $T_{j}$; la contrainte (3.13) force l'échéance à être supérieure aux intérêts dus, plus l'amortissement mensuel minimum $C_{\min }$. 
Ce modèle est ensuite allégé en s'appuyant sur les propriétés précédemment démontrées. Les durées de chaque prêt $P$ sont filtrées en ne conservant que les durées multiples de 12 mois, c'est-à-dire s'exprimant en années. Sont ajoutées à cet ensemble les durées (non multiples de 12) correspondant aux dates de variation de taux dans la grille ou de variation de capacité de remboursement (Lem. 3.4). En pratique, le nombre de variables $d_{j}$ reste en $O(G)$ (avec $G$ le nombre de plages de durées des grilles de taux). De plus, l'approximation qui est faite dans le cas où le prêt $P$ est le plus long du plan est sans conséquence sur la qualité de la solution obtenue; nous observons que la structure du plan obtenu en pratique reste identique à celle du plan optimal. Ensuite, les coupes définies par le Lemme 3.3 sont introduites dans le programme. Pour toute paire de variables $d_{j}, d_{j^{\prime}}^{\prime}$ (associées respectivement aux prêts $P$ et $P^{\prime}$ ) avec $j^{\prime} \leq j$ telle que la capacité de remboursement $\bar{E}$ en $j^{\prime}$ est la même qu'en $j$ et le taux du prêt $P^{\prime}$ en $j^{\prime}$ est supérieur au taux du prêt $P$ en $j$, nous posons la contrainte $d_{j^{\prime}}^{\prime} \leq 1-d_{j}$. L'ensemble des coupes peut être généré en temps $O\left(n^{2} G^{2}\right)$.

Nous ne nous étendrons pas d'avantage sur les propriétés et la modélisation des prêts à profil constant, en voie de disparition dans le secteur libre. En revanche, nous y reviendrons dans le cadre de la modélisation des prêts réglementés épargne logement (PEL, CEL).

\subsection{LES PRÊTS À PROFIL LIBRE}

Un prêt à profil libre se caractérise par son montant $m$, une durée $d$ et $r$ paliers $\left(d_{k}, e_{k}\right)$ avec $\sum_{k=1}^{r} d_{k}=d$. L'équation (3.9) peut alors être étendue pour décrire l'amortissement sur $r$ paliers :

$$
m=\sum_{k=1}^{r} e_{k} \cdot \operatorname{VAN}\left(a_{k}, b_{k}, T_{d}\right)
$$

où $a_{k}=1+\sum_{k^{\prime}=1}^{k-1} d_{k^{\prime}}\left(\right.$ resp. $\left.b_{k}=a_{k}+d_{k}-1\right)$ représente la date de début (resp. fin) du palier $k$. Lorsque la durée de chaque palier est fixée, le taux l'étant lui aussi, l'équation (3.14) devient linéaire en le montant $m$ et les échéances $e_{k}$.

\subsubsection{Propriétés et complexité}

Contrairement aux prêts à profil constant, la présence d'au moins un prêt à profil libre au sein d'un plan de financement garantit le bon emploi de la capacité de remboursement. En effet, nous pouvons faire l'observation suivante.

Observation 3.6. Soit un plan de financement optimal composé d'un prêt à profil libre $P$ de durée $d$ et durée minimum $D_{\min }$ :

(a) si le plan sature la capacité de remboursement en $j<d$, alors il sature le capacité du premier mois jusqu'au mois $j$;

(b) si le plan sature la capacité de remboursement en $D_{\min }$, alors il sature le capacité du premier mois jusqu'au mois $d-1$. 
Démonstration. Admettons que l'assertion (a) soit fausse et notons $j^{\prime}<j$ un mois où la capacité n'est pas saturée. En augmentant le capital amorti du prêt $P$ au mois $j^{\prime}$ tout en maintenant sa durée $d$ (ce qui est faisable en abaissant le montant de sa dernière échéance), nous réduisons le coût du plan, ce qui contredit l'optimalité de ce dernier. La preuve de l'assertion (b) découle des mêmes arguments.

Sauf mention contraire et jusqu'à la fin de cette section, nous considérerons les prêts étudiés comme à profil libre et sans contrainte de montant. Tout d'abord, nous montrons comment borner efficacement la durée des prêts qui composent un plan de financement optimal. Pour cela, nous définissons pour chaque prêt $P$ la durée $d_{P}^{*}$ du plan optimal $\mathcal{P}_{P}$ composé uniquement de $P$ (lorsque qu'un tel plan existe, c'est-à-dire lorsque $d_{P}^{*} \leq D_{\max }$ ).

Lemme 3.7. Unique lorsque le plan $\mathcal{P}_{P}$ existe, la durée $d_{P}^{*}$ peut être calculée en temps $O\left(D_{\max } \log G\right)$.

Démonstration. Tout d'abord, nous démontrons l'unicité de la durée $d_{P}^{*}$. Soit $\mathcal{P}_{P}$ le plan optimal de durée $d_{P}^{*} \geq D_{\min }$ minimale. S'il existe un autre plan optimal d'une durée $d>d_{P}^{*}$, celui-ci doit saturer la capacité de remboursement $E_{j}$ pour tout $j=1, \ldots, d-1$. En effet, si tel n'est pas le cas, un plan de coût moindre peut être obtenu en augmentant le capital amorti d'une échéance $j<d$ non saturée (et ce même en cas de diminution de la durée, puisque la grille de taux est croissante), ce qui est une contradiction. Nous en déduisons que ce plan a un coût strictement supérieur à $\sum_{j=1}^{d-1} E_{j}-B$ et donc à $\sum_{j=1}^{d_{P}^{*}} E_{j}-B$ puisque $d>d_{P}^{*}$. Or, d'après notre première hypothèse, cette dernière expression est une borne supérieure du coût du plan $\mathcal{P}_{P}$. Par conséquent, ce plan de durée $d>d_{P}^{*}$ ne peut être optimal, ce qui contredit son existence même.

La durée $d_{P}^{*}$ peut être calculée par dichotomie sur l'ensemble des plages de taux de la grille, chaque itération consistant à dérouler les équations d'amortissement (1.1)-(1.4) pour un montant $B$ et des échéances $E_{j}$, de manière à obtenir la durée du plan pour le taux de la plage considérée. Si la durée obtenue est supérieure (resp. inférieure) à la durée maximale (resp. minimale) de la plage considérée, une nouvelle itération avec le taux d'une plage supérieure (resp. inférieure) est nécessaire; lorsque cette durée se situe dans la plage, elle correspond à la durée $d_{P}^{*}$ recherchée. Deux cas particuliers se présentent : si la durée obtenue avec le taux le plus bas (resp. haut) de la grille est inférieure (resp. supérieure) à $D_{\min }$ (resp. $\left.D_{\text {max }}\right)$, alors $d_{P}^{*}=D_{\text {min }}\left(\right.$ resp. il n'existe pas de plan $\left.\mathcal{P}_{P}\right)$.

Proposition 3.8. Soit $d^{*}$ la plus petite des durées $d_{P}^{*}$. Tout plan de financement optimal (et donc tout prêt qui le compose) a une durée inférieure à $d^{*}$, qui peut être calculée en temps $O\left(n D_{\max } \log G\right)$.

Démonstration. Admettons qu'un plan optimal contienne le prêt $P$ d'une durée $d>d^{*} \geq D_{\min }$. D'après les mêmes arguments que ceux employés précédemment, ce plan doit saturer la capacité $E_{j}$ pour tout $j=1, \ldots, d-1$, sans quoi il ne peut être optimal. Nous en déduisons que celui-ci a un coût strictement supérieur à $\sum_{j=1}^{d-1} E_{j}-B$ et donc à $\sum_{j=1}^{d^{*}} E_{j}-B$. Or, cette dernière expression est une borne 


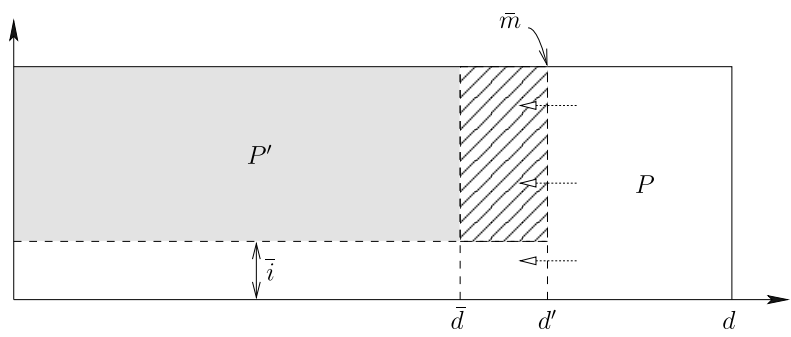

Figure 4. L'emboîtement optimal des prêts $P$ et $P^{\prime}$.

supérieure du coût d'un plan optimal, ce qui contredit notre première hypothèse.

À présent, nous étudions la structure d'un plan de financement optimal (à base prêts à profil libre, sans contrainte de montant). Voici une règle de dominance plus forte que celle énoncée dans le Lemme 3.3 pour les prêts à profil constant.

Lemme 3.9 (dominance). Si un prêt $P$ à taux $t$ appartient à un plan de financement optimal et a comme date de fin $d$, alors ce dernier ne peut contenir un prêt $P^{\prime}$ à profil libre (ou constant) de taux $t^{\prime}>t$ ayant comme date de fin $d^{\prime} \leq d$.

Démonstration. Admettons qu'un plan optimal contienne un tel prêt $P^{\prime}$. Le prêt $P$ ayant un montant maximum supérieur au besoin de financement $B$, le montant du prêt $P^{\prime}$ peut être transféré sur le prêt $P$ à un taux moindre, ce qui permet d'abaisser le coût du plan (et ce même en cas de diminution de la durée de $P$, puisque les grilles de taux sont croissantes).

À travers la discussion suivante, nous montrons que les prêts composant un plan de financement optimal possèdent une structure singulière. Afin d'éviter de trop lourds détails techniques, nous considérons dans un premier temps que le montant d'amortissement mensuel minimum des prêts est nul $\left(C_{\min }=0\right)$.

Soit un plan de financement composé d'un prêt $P$ à profil libre de taux $t$. Ce prêt $P$ possède une durée $d$, un montant $m$ et des échéances $e_{1}, \ldots, e_{d}$. Supposons qu'il existe un prêt $P^{\prime}$ dont la grille de taux autorise un emprunt à taux $t^{\prime}<t$ sur une durée $d^{\prime} \leq d$. À présent, notons $\bar{m}$ le capital restant dû à l'issue du mois $d^{\prime}$ sur le prêt $P$ et $\bar{i}$ le montant mensuel des intérêts à verser pour un tel capital, soit $t \cdot \bar{m}$. Le prêt $P$ reste valide si l'on redéfinit son montant comme étant égal à $\bar{m}$ et ses $d^{\prime}$ premières échéances à $\bar{i}$. Le montant $m-\bar{m}$, actuellement amorti au taux $t$ via les échéances $e_{1}-\bar{i}, \ldots, e_{d^{\prime}}-\bar{i}$, peut alors être emprunté sur le prêt $P^{\prime}$ au taux $t^{\prime}$ (puisque sur $d^{\prime}$ mois). L'amortissement se faisant avec un taux moindre, celui-ci sera de coût inférieur, offrant par là-même un résidu de capacité de remboursement à partir du mois $\bar{d} \leq d^{\prime}$ (voir Fig. 4). Ce résidu peut aussitôt être exploité afin de réduire la durée de $P$ de $d^{\prime}-\bar{d}$ mois, permettant de réduire encore le coût du plan.

À présent, l'amortissement du montant $\bar{m}$ du prêt $P$ débute, non plus à partir du mois $d^{\prime}$, mais à partir du mois $\bar{d}$. Par conséquent, le processus décrit précédemment 


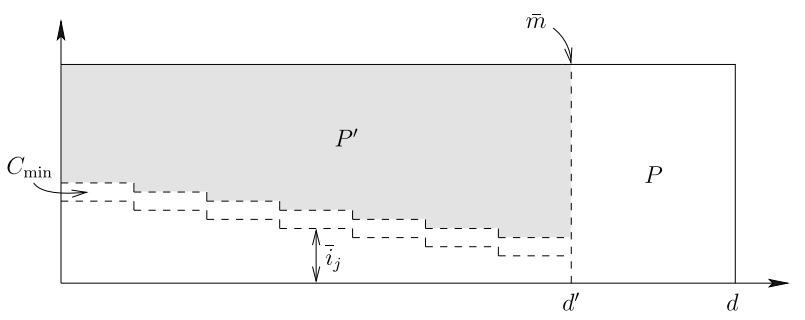

Figure 5. L'emboîtement optimal des prêts $P$ et $P^{\prime}$ lorsque $C_{\min }>0$.

peut être réitéré en posant $m=\bar{m}$ et en notant à nouveau $\bar{m}$ le capital restant dû à l'issue du mois $d^{\prime}$ (qui est donc strictement inférieur à celui de l'étape précédente). Le montant dégagé du prêt $P$ peut être reporté sans incidence sur le prêt $P^{\prime}$, du fait du caractère additif des équations d'amortissement à taux égal. Ce processus converge puisque la valeur de $\bar{m}$ décroît strictement à chaque nouvelle étape, tendant vers zéro, alors que la valeur de $\bar{d}$ tend vers $d^{\prime}$, le coût des plans successivement obtenus diminuant lui aussi. Il est à noter que le plan terminal peut n'être plus composer que du prêt $P^{\prime}$, lorsque la totalité du montant du prêt $P$ a été reporté sur $P^{\prime}$.

Ce raisonnement peut être généralisé dans le cas où l'amortissement mensuel minimum $C_{\min }$ n'est pas nul. Il suffit de poser $\bar{m}$ comme étant le capital restant dû à l'issue du mois $d^{\prime}$ auquel on ajoute le montant $d^{\prime} \cdot C_{\min }$. Dès lors, le prêt $P$ reste valide si l'on redéfinit son montant comme étant égal à $\bar{m}$ et ses $d^{\prime}$ premières échéances à $\bar{i}_{1}+C_{\min }, \ldots, \bar{i}_{d^{\prime}}+C_{\min }$, où $\bar{i}_{j}$ correspond aux intérêts dus au mois $j$, soit $t\left(\bar{m}-(j-1) C_{\min }\right)$ (voir Fig. 5$)$.

En présence de plusieurs prêts, la proposition suivante peut être établie.

Proposition 3.10. Tout plan de financement optimal composé seulement de prêts à profil libre sans contrainte de montant satisfait les deux assertions suivantes :

(a) l'ordre induit par les taux des prêts du plan est le même que l'ordre induit par les durées des prêts;

(b) hormis la durée du prêt le plus long, les durées des prêts coïncident avec des dates de variation de taux dans la grille.

Le lecteur notera que cette proposition est valide quel que soit le profil de la capacité de remboursement contrairement à la Proposition 3.5 concernant les prêts à profil constant.

\subsubsection{Modélisation par contraintes linéaires mixtes}

Dans cette section, nous décrivons comment sont modélisés les prêts à profil libre au sein du programme linéaire mixte. Nous nous appuyons sur la Proposition 3.10 pour filtrer l'ensemble des contraintes régissant l'amortissement d'un prêt à profil libre, permettant ainsi une résolution en temps réel. 
Un filtrage de premier niveau concerne les variables booléennes $d_{j}$, à 1 si la durée du prêt $P$ est $j$ et à 0 sinon. En nous appuyant sur la proposition précédente, nous ne conservons que les variables $d_{j}$ correspondant à une date de variation de taux dans la grille de $P$ inférieure à la durée $d^{*}$. Il nous faut ensuite décrire l'amortissement du prêt jusqu'à chacune de ces durées $d_{j}$. Un filtrage de second niveau consiste alors à supposer que l'amortissement jusqu'à la durée $j$ se fait par paliers entre un ensemble de dates remarquables : les dates de variation de taux dans la grille ou de variation de capacité de remboursement, auxquelles on ajoute les dates multiples de 12 mois afin de renforcer le maillage.

L'amortissement jusqu'à la durée $j$ est décrit à l'aide de la contrainte suivante, caractérisant le capital restant dû à l'issu du palier $k$ de durée $D_{j, k}$ :

$$
\operatorname{crd}_{j, k}=\operatorname{crd}_{j, k-1}-\alpha\left(D_{j, k}, T_{j}\right) \cdot\left(e_{j, k}-T_{j} \cdot \operatorname{crd}_{j, k-1}\right)
$$

où le coefficient

$$
\alpha(d, t)=\sum_{r=0}^{d-1}(1+t)^{r}=\frac{(1+t)^{d}-1}{t}
$$

décrit l'évolution de la part de capital amorti à échéance constante sur une durée $d$ au taux $t$. Ce coefficient s'obtient à partir des équations d'amortissement (1.1)(1.2) en considérant $e_{1}=\cdots=e_{j}$. Ces contraintes doivent être complétées en posant $\operatorname{crd}_{j, 0}=m_{j}$ (le capital restant dû en début de prêt correspond au montant emprunté) et $\operatorname{crd}_{j, K}=0$ où $K$ est l'indice du dernier palier d'amortissement (le palier ayant comme date de fin $j$ ), ainsi que les contraintes garantissant le remboursement des intérêts et de l'amortissement mensuel minimum, soit :

$$
e_{j, k} \geq T_{j} \cdot \operatorname{crd}_{j, k-1}+C_{\min } \cdot d_{j}
$$

Ces contraintes forcent l'amortissement à se dérouler jusqu'à la date $j$, ce qui n'est pas exact si le prêt en question est le plus long du plan de financement. Dans ce cas précis, nous observons en fin de prêt un amortissement résiduel d'un montant légèrement supérieur à $C_{\text {min }}$, signifiant que le prêt aurait pu être complètement amorti à la date $\bar{j}$ mais que la contrainte à forcer l'amortissement à se poursuivre jusqu'en $j$. Ce résidu sera gommé dans la dernière phase de l'heuristique de résolution.

Comme nous l'avons vu dans la discussion précédente, l'emboîtement optimal de deux prêts à profil libre à l'amortissement mensuel minimum strictement positif fait apparaître autant de paliers que ce qu'il y a de mois (Fig. 5), ce qui n'est pas acceptable du point de vue marketing et commercial. De plus, une multitude de paliers engendre un surcoût de traitement non négligeable dans la chaîne aval de gestion (en particulier, la modification de la mensualité prélevée automatiquement sur le compte du client). Afin de pouvoir maîtriser le nombre de paliers d'un prêt à profil libre, nous intégrons dans la fonction objectif du programme un terme visant à limiter le nombre de paliers du prêt (ce nombre peut également être contraint si besoin est). Le nombre de paliers est compté grâce à des variables booléennes $p_{k}$ 
prenant la valeur 1 lorsque les échéances $e_{k-1}$ et $e_{k}$ sont différentes, à l'aide des contraintes suivantes :

$$
\begin{aligned}
& p_{j, k} \geq\left(e_{j, k-1}-e_{j, k}\right) / E_{\max } \\
& p_{j, k} \geq\left(e_{j, k}-e_{j, k-1}\right) / E_{\max }
\end{aligned}
$$

où $E_{\max }$ est une constante supérieure à la variation maximale de l'échéance au cours du plan (dans le cas où la capacité du client est donnée, il suffit de choisir $E_{\max }$ comme la capacité maximale de remboursement du client).

Enfin, les coupes définies par le Lemme 3.9 sont ajoutées au programme. Pour toute paire de variables $d_{j}, d_{j^{\prime}}^{\prime}$ (associées respectivement aux prêts $P$ et $P^{\prime}$ ) avec $j^{\prime} \leq j$ telle que le taux du prêt $P^{\prime}$ en $j^{\prime}$ est supérieur au taux du prêt $P$ en $j$, nous posons la contrainte $d_{j^{\prime}}^{\prime} \leq 1-d_{j}$. Comme pour les prêts à profil constant, l'ensemble des coupes peut être généré en temps $O\left(n^{2} G^{2}\right)$.

\section{LES PRÊTS RÉGLEMENTÉS}

Les prêts du secteur réglementé possèdent généralement des caractéristiques singulières, nous obligeant à adopter une modélisation et un traitement particulier pour chacun. Nous avons eu à traiter six types de prêts réglementés : le prêt plan épargne logement (PEL), le prêt compte épargne logement (CEL), le prêt à $0 \%(\mathrm{PTZ})$, le prêt Paris logement (PPL), le prêt conventionné $(\mathrm{PC})$, le prêt à l'accession sociale (PAS). La réglementation de ces prêts, modifiée par décrets, est publiée dans le Journal officiel et peut être consultée sur le site du Ministère du logement [12].

\subsection{LES PRÊTS CONVENTIONNÉS}

Le prêt conventionné (PC) et son dérivé, le prêt à l'accession sociale (PAS), peuvent être modélisés comme des prêts à profil libre. Ces prêts ont pour vocation à couvrir une grande partie du besoin de financement à des taux préférentiels (subventionnés par l'État français), notamment le PAS qui n'est accessible que sous conditions de ressources. D'un autre côté, ceux-ci ne sont compatibles au sein du plan de financement qu'avec certains prêts réglementés (entre autres, le PEL et le PTZ). Nous ne nous attarderons donc pas d'avantage sur ces deux types de prêts.

\subsection{LES PRÊTS ÉPARGNE LOGEMENT}

Le plan épargne logement (PEL) et le compte épargne logement (CEL) sont des produits financiers originaux, qui comptent parmi les placements préférés des français. Leur originalité réside dans le fait qu'ils comportent deux phases : une première phase d'épargne rémunérée à taux fixe (relativement attractive car exonérée d'impôt sur le revenu), puis la possibilité d'obtenir un prêt immobilier à un taux fixe déterminé par les dates de versement à l'épargne. La durée du prêt, 
annuelle, peut varier entre 2 et 15 ans et son montant est calculé de façon à ce qu'à échéance constante, les intérêts dus durant la phase de prêt soient couverts par les intérêts perçus durant la phase d'épargne (auxquels s'ajoute une prime d'État). L'emprunteur bénéficie donc du même taux quelque soit la durée de son emprunt, mais en contrepartie, le montant qu'il peut emprunter décroît à mesure que cette durée s'allonge.

Soit $I$ les droits acquis à l'issue de la phase d'épargne et $T$ le taux d'intérêt de la phase de prêt. Pour une durée $d$, l'échéance $e=m / \operatorname{VAN}(1, d, T)$ du prêt doit satisfaire $e \cdot d-m \leq I$, impliquant que le montant $m$ soit borné par l'expression

$$
\frac{I}{d / \operatorname{VAN}(1, d, T)-1}
$$

dont la valeur décroît à mesure que $d$ augmente (en effet, le ratio $d / \operatorname{VAN}(1, d, T)$ est d'autant plus petit que $d$ est petit). Si nous en restions là, la modélisation par contraintes linéaires mixtes des prêts épargne logement ne serait donc pas plus complexe que celle des prêts du secteur libre à profil constant, soit pour toute durée admissible $j$ :

$$
\begin{array}{r}
m_{j} \leq d_{j} \cdot M_{\text {max }_{j}} \\
m_{j}=e_{j} \cdot \operatorname{VAN}(1, j, T)
\end{array}
$$

avec $M_{\text {max }_{j}}=\frac{I}{j / \operatorname{VAN}(1, j, T)-1}$. Cependant, ce simple modèle ne suffit pas à couvrir tous les points de la longue réglementation de l'épargne logement.

\subsubsection{Les prêts plan épargne logement (PEL)}

Dans le cas du PEL, le taux d'emprunt du prêt est fixé dès l'ouverture du plan. Lorsque le prêt n'est composé que d'un plan, le modèle précédent est donc utilisable directement. Cependant, bien qu'une personne ne puisse ouvrir qu'un seul PEL, un prêt épargne logement peut dans certains cas être composé à partir de plusieurs plans : l'emprunteur possède des plans qui lui ont été cédés par des membres de sa famille ou les deux emprunteurs forment un couple marié. Les plans sont alors agrégés pour ne former qu'un seul prêt (nous verrons par la suite que cela est avantageux vis-à-vis du coût des garanties). Si un montant $m_{k}$ est emprunté au taux $T_{k}$ sur chaque plan $k$, l'échéance $e$ du prêt agrégé sur une durée $d$ est calculé à partir d'un taux moyen $T$, comme suit :

$$
e=\frac{\sum_{k} m_{k}}{\operatorname{VAN}(1, d, T)} \quad T=\frac{\sum_{k} m_{k} \cdot T_{k}}{\sum_{k} m_{k}} .
$$

Ce calcul, sans véritable sens financier mais toujours en vigueur dans la chaîne aval de gestion, est l'héritage de pratiques anciennes destinées à simplifier le calcul de l'échéance en l'absence d'ordinateur. En toute logique, l'échéance du prêt devrait être :

$$
e=\sum_{k} \frac{m_{k}}{\operatorname{VAN}\left(1, d, T_{k}\right)} .
$$


La contrainte (4.22) n'étant pas linéaire, nous avons justement choisi de l'approcher par la contrainte (4.23) dans notre modèle linéaire mixte. En notant $T_{\min }=\min _{k} T_{k}$ et $T_{\max }=\max _{k} T_{k}$, l'erreur absolue entre l'échéance réelle et l'échéance approchée peut être bornée par l'expression

$$
\sum_{k} m_{k} \cdot\left(\frac{1}{\operatorname{VAN}\left(1, d, T_{\min }\right)}-\frac{1}{\operatorname{VAN}\left(1, d, T_{\max }\right)}\right)
$$

puisque le taux moyen $T$ est compris, comme tout $T_{k}$, entre $T_{\min }$ et $T_{\max }$ et que la fonction VAN est croissante à durée fixée. En pratique, l'écart constaté est très faible, inférieur à l'euro, rendant l'approche viable. Considérons par exemple deux plans permettant un emprunt de 70000 euros à $6.32 \%$ sur 15 ans pour le premier (ouvert avant 1994) et un emprunt de 10000 euros sur la même durée à $4.20 \%$ pour le second (ouvert après 2004) ; l'échéance approchée est de 677.46 euros alors que l'échéance réelle est de 677.85 euros.

Pour un prêt PEL composé à partir de plans acquis $(A)$ et cédés $(C)$, le modèle générique sera donc le suivant :

$$
\begin{aligned}
\sum_{j \in\{24,36, \ldots, 180\}} d_{j} & =p \\
\sum_{k \in A \cup C} d_{j, k} & =d_{j} \quad \forall j \\
e_{j} & =\sum_{k \in A \cup C} m_{j, k} / \operatorname{VAN}\left(1, j, T_{j, k}\right) \quad \forall j \\
d_{j, k} \cdot M_{\min _{j, k}} & \leq m_{j, k} \leq d_{j, k} \cdot M_{\max _{j, k}} \quad \forall j, \forall k
\end{aligned}
$$

auquel il faut ajouter les contraintes

$$
\begin{aligned}
& \sum_{k \in C} d_{j, k} \leq d_{j, k} \quad \forall j, \forall k \in A \\
& \sum_{k \in C} d_{j, k} \cdot \sum_{k \in A} M_{\max _{j, k}}=\sum_{k \in A} m_{j, k} \quad \forall j
\end{aligned}
$$

forçant les droits acquis à être consommés avant les droits cédés (les inégalités (4.28) sont ajoutées pour renforcer la relaxation linéaire du programme).

\subsubsection{Les prêts compte épargne logement (CEL)}

Le cas du prêt CEL est compliqué par le fait que le taux du prêt n'est pas seulement fonction de la date d'ouverture du compte, mais dépend aussi des dates (communément appelées tranches) auxquelles sont effectués les versements. Après révision du taux par décret (c'est-à-dire création d'une nouvelle tranche), tout versement futur sera soumis au nouveau taux en vigueur, contrairement au PEL. Le taux d'emprunt du prêt pour une durée donnée s'obtient en faisant la moyenne des taux des tranches pondérés par les montants empruntés sur chacune (y compris 
lorsque le prêt est composé à partir de plusieurs comptes). Ainsi, un CEL peut être vu comme une succession de "petits" PEL, avec une contrainte supplémentaire qui est que les droits les plus anciens doivent être consommés avant les plus récents (de même que les droits acquis doivent toujours être consommés avant les droits cédés).

Le taux minimum (resp. maximum) d'emprunt rencontré jusqu'à présent pour une tranche est de $2.75 \%$ (resp. $4.25 \%$ ). Par conséquent, nous pouvons décliner le modèle exposé précédemment pour les PEL, la consommation des droits par ordre d'ancienneté étant assurée par l'addition des contraintes

$$
\begin{aligned}
& d_{j, k, 1} \leq d_{j, k, 2} \leq \cdots \leq d_{j, k, t} \quad \forall j, \forall k \\
& \sum_{t^{\prime}>t} d_{j, k, t^{\prime}} \cdot M_{\max _{j, k, t}}=m_{j, k, t} \quad \forall j, \forall k
\end{aligned}
$$

où l'indice $t$ dénote le numéro de la tranche concernée, tel que toute tranche $t^{\prime}<t$ précède la tranche $t$ (les inégalités (4.30) sont ajoutées pour renforcer la relaxation linéaire du programme).

Enfin, l'encours maximum d'un PEL (resp. CEL) est de 92000 (resp. 23000) euros; l'encours PEL et CEL confondus est également limité à 92000 euros. L'encours minimum d'un PEL ou d'un CEL est de 155 euros. Ces bornes sont à vérifier par opération, mais également par emprunteur et par bien immobilier objet de l'opération lorsque ceux-ci interviennent dans des opérations extérieures. Ces contraintes peuvent être ajoutées aux précédentes sans détériorer le caractère linéaire du modèle.

\subsection{LES PRÊTS À TAUX ZÉRO}

Les prêts à taux zéro (PTZ, PPL) sont sans doute les prêts aidés les plus réglementés, puisqu'ils n'engagent à aucun versement d'intérêts aux créanciers. Ces prêts "gratuits" sont naturellement destinés aux personnes aux revenus les plus modestes, d'où la réglementation stricte les encadrant. Ainsi, de nombreuses conditions sont requises pour en bénéficier; ces conditions portent entre autres sur l'objet du financement, le type de bien financé, la destination du bien, mais surtout sur les bénéficiaires et leurs ressources. Les caractéristiques financières des prêts à taux zéro sont elles aussi très strictes : le montant de l'emprunt est faible et le profil du prêt quasiment figé. De plus, certaines contraintes sont imposées au plan de financement les contenant. Dans cette section, nous détaillons quelles sont les contraintes qui importent dans le cadre de l'optimisation du plan de financement et comment nous les avons traitées.

\subsubsection{Le prêt Paris logement (PPL)}

Le montant du PPL doit être inférieur à un montant maximum déterminé par le nombre de personnes occupant le bien financé ; le PPL a une durée fixe de 15 ans sur laquelle le montant doit être remboursé à échéance constante. La présence d'un PPL au sein du plan de financement ne peut se faire qu'à la condition suivante : 
le prix d'achat du logement doit être financé pour au moins $50 \%$ de sa valeur par des prêts de plus de 15 ans.

Ainsi, le PPL est décrit au sein du modèle linéaire mixte par les contraintes

$$
\begin{aligned}
m_{\mathrm{PPL}} & \leq p_{\mathrm{PPL}} \cdot M_{\max _{\mathrm{PPL}}} \\
e_{\mathrm{PPL}} & =m_{\mathrm{PPL}} / D_{\mathrm{PPL}} \\
\sum_{i \neq \mathrm{PPL}} \sum_{j \geq D_{\mathrm{PPL}}} m_{i, j} & \geq p_{\mathrm{PPL}} \cdot B_{\mathrm{PPL}}
\end{aligned}
$$

où $D_{\mathrm{PPL}}$ est la durée fixe du PPL (actuellement $D_{\mathrm{PPL}}=180$ mois) et $B_{\mathrm{PPL}}$ une constante dérivée du besoin de financement (ici le prix d'achat du logement plus certains frais). Afin de faciliter l'accès au PPL, la contrainte (4.34) a récemment été retirée de la réglementation.

\subsubsection{Le prêt à $0 \%$ Ministère du logement (PTZ)}

Dans le cas du PTZ, deux contraintes nous intéressent dans le cadre de l'optimisation du plan de financement. La première porte sur son montant. En effet, la réglementation définit trois plafonds que le montant du prêt ne doit pas dépasser. Le premier plafond est déterminé par le nombre de personnes occupant le bien financé et par la localisation de celui-ci (Île-de-France ou province). Le deuxième plafond est égal à un pourcentage (actuellement $30 \%$ si le logement est en zone franche, $20 \%$ sinon) d'un montant dérivé du besoin de financement. Enfin, le troisième plafond est égal à une fraction (actuellement un tiers) d'un montant dérivé du besoin de financement et diminué de la somme des montants des prêts du plan de financement ayant une durée inférieure ou égale à 2 ans. Du fait de ce dernier plafond, le montant du PTZ dépend, dans une certaine mesure, des montants des autres prêts du plan de financement. De façon générale, cette contrainte de montant s'écrit comme suit :

$$
\begin{aligned}
& m_{\mathrm{PTZ}} \leq p_{\mathrm{PTZ}} \cdot M_{\text {maxPTZ }} \\
& m_{\mathrm{PTZ}} \leq \frac{1}{3}\left(B_{\mathrm{PTZ}}-\sum_{\forall i \neq \mathrm{PTZ}} \sum_{\forall j \leq 24} m_{i, j}\right)
\end{aligned}
$$

où $M_{\text {max }}$ Pтz représente le minimum des deux premiers plafonds et $B_{\text {PTZ }}$ est la constante dérivée du besoin de financement sur laquelle est basée le calcul du troisième plafond.

La seconde contrainte porte sur la durée du plan de financement. Le nombre de paliers de remboursement du PTZ (1 ou 2) ainsi que leurs durées sont déterminées par les ressources de l'emprunteur. En sus, nous avons la contrainte suivante : dans le cas où le PTZ comporte deux paliers, la durée du premier doit être inférieure à la durée du prêt le plus long du plan de financement (hors PTZ). Si ce n'est pas le cas, la durée du premier palier du PTZ peut être raccourcie, tout en restant supérieure à 72 mois. Cette contrainte, purement combinatoire, est délicate à 
intégrer en l'état dans le modèle linéaire mixte. De plus, elle peut théoriquement empêcher l'existence d'une solution (par exemple, si le plan ne contient que des prêts complémentaires de type épargne logement). En effet, toute réduction de la durée du premier palier du PTZ induit une augmentation du montant de son échéance (puisque le capital amorti sur chaque palier est fixé), ce qui se traduit par un abaissement de la capacité de remboursement sur les prêts complémentaires et donc un allongement de leur durée. Or cet allongement peut entraîner le durcissement de certaines contraintes de prêt (montant maximum plus bas, taux d'emprunt plus cher), conduisant à une absence de solution.

Toutefois, en pratique, les plans de financement des personnes ayant droit au PTZ sont naturellement longs (puisqu'ayant de faibles revenus, ils ont en toute logique une faible capacité de remboursement); une exception typique est le cas du jeune cadre dont les revenus $N-2$ sont nuls et les revenus actuels élevés. Aussi nous avons pris le parti de traiter cette contrainte en deux temps, de manière spécifique, afin de parvenir à une résolution en temps réel. Tout d'abord, le PTZ est intégré au programme linéaire mixte sous sa forme prévue initialement par la réglementation : $e_{1}=\left(R_{1} \cdot m\right) / D_{1}$ et $e_{2}=\left(R_{2} \cdot m\right) / D_{2}$ où $R_{1}, R_{2}$ sont les ratios de capital à amortir sur chacun des paliers et $D_{1}, D_{2}$ les durées de ces derniers. Si ce programme n'a pas de solution, alors nous pouvons conclure que le problème n'admet aucune solution (puisque le calcul a été réalisé avec le profil du PTZ le plus favorable, celui dont l'amortissement est le plus tardif). Si ce programme admet une solution, alors nous notons $d_{\text {inf }}$ la durée du prêt le plus long du plan obtenu (hors PTZ); si ce prêt est à profil libre, alors $d_{\text {inf }}$ est choisie comme la date remarquable à partir duquel l'amortissement résiduel $C_{\min }$ apparaît. Dès lors, deux cas de figure se présentent. Si $d_{\text {inf }}$ est supérieure ou égale à $D_{1}$, la durée initiale du premier palier du PTZ, alors le plan obtenu respecte la contrainte et par conséquent est optimal. Dans le cas contraire $\left(d_{\text {inf }}<D_{1}\right)$, l'observation suivante est immédiate.

Observation 4.1. La durée du prêt le long (hors PTZ) de tout plan admissible (s'il en existe un) est supérieure à $d_{\text {inf }}$.

Dans ce cas, la recherche d'un plan optimal se fait en résolvant un second programme dans lequel la durée du premier palier du PTZ devient variable, représentée par des booléens $d_{\mathrm{PTZ}, j}$ en exclusion mutuelle, avec $j \in\left\{d_{\text {inf }}^{\prime}, \ldots, D_{1}\right\}$ (où $d_{\text {inf }}^{\prime}=$ $\max \left\{d_{\text {inf }}, 72\right\}$, la durée du premier palier du PTZ ne pouvant être réduite en deçà de 72 mois). Notons que seules les durées correspondant aux dates remarquables dans cet intervalle sont conservées. Afin d'assurer la validité du plan, il suffit alors de poser pour chaque variable $d_{\mathrm{PTZ}, j}$ la contrainte

$$
\sum_{\forall i \neq \mathrm{PTZ}} \sum_{\forall j^{\prime} \geq j} d_{i, j^{\prime}} \geq d_{\mathrm{PTZ}, j}
$$

garantissant qu'au moins un prêt du plan (autre que le PTZ) aura une durée $j^{\prime}$ supérieure ou égale à la durée $j$ du premier palier du PTZ.

Dans le cas où le prêt le long du plan est à profil libre, un plan optimal aura le profil décrit sur la Figure 6. En effet, l'intérêt économique du client est que 


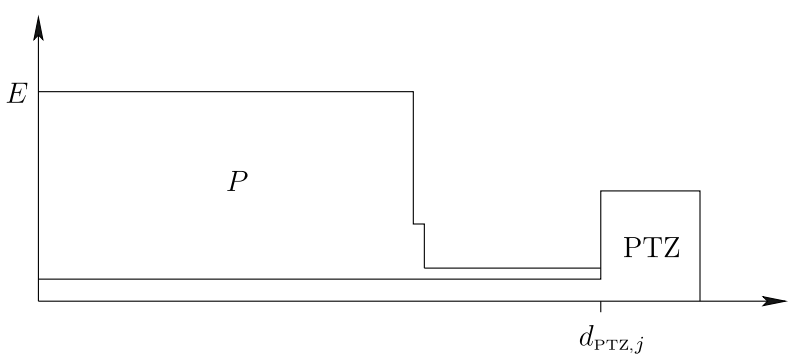

Figure 6. PTZ + prêt à profil libre : plan optimal.

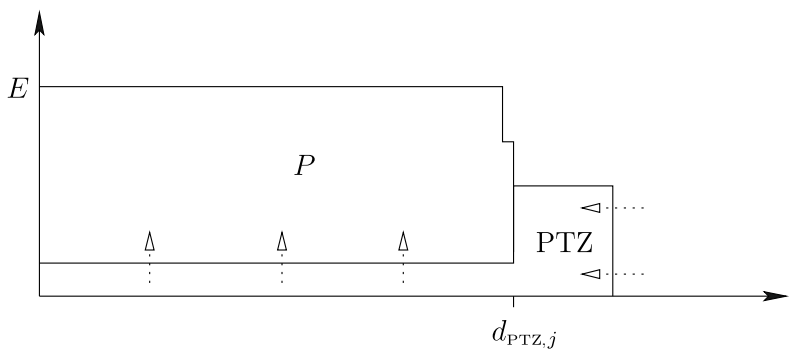

Figure 7. PTZ + prêt à profil libre : plan idéal.

l'amortissement du PTZ soit le plus tardif possible (puisqu'il ne lui coûte rien). Par conséquent, en ramenant seulement la durée du premier palier du PTZ à la durée maximale pour laquelle le taux de la grille du prêt à profil libre ne change pas, nous obtenons le meilleur montage. Toutefois, pour des raisons commerciales, une solution minimisant la durée totale du plan de financement sera préférée ici (voir Fig. 7), la différence en terme de coût étant minime. En pratique, la durée "idéale" du premier palier du PTZ est proche de la durée $d_{\mathrm{inf}}$. Ainsi, la résolution de ce second programme peut être accélérée en branchant sur les variables $d_{\mathrm{PTZ}, j}$ dans l'ordre croissant des durées $j$, la première solution admissible trouvée étant retournée.

\section{Assurances, garanties et autres frais}

Dans cette section, nous voyons comment intégrer efficacement assurances et garanties dans le modèle linéaire mixte décrit précédemment.

\subsection{Assurances}

Dans le cas d'une assurance sur capital restant dû (CRD), le taux $A$ de l'assurance vient simplement s'ajouter au taux du prêt dans les équations d'amortissement. Par conséquent, les modèles exposés dans les Sections 3.1.2 et 3.2.2, ainsi 
que toutes les propriétés énoncées, restent valables à condition de considérer les taux des prêts assurances comprises, les échéances devenant de fait des échéances assurances comprises.

Le cas avec assurance sur capital initial (CI) est moins évident. La prime d'assurance à verser mensuellement pour un prêt de montant $m$ est égale à $A \cdot m$. Ainsi, pour un prêt à profil constant, les contraintes (3.12) et (3.13) décrivant l'amortissement du prêt sur une durée $j$ deviennent :

$$
\begin{aligned}
& e_{j}=\left(A+1 / \operatorname{VAN}\left(1, j, T_{j}\right)\right) \cdot m_{j} \\
& e_{j} \geq\left(T_{j}+A\right) \cdot m_{j}+C_{\min } \cdot d_{j} .
\end{aligned}
$$

Pour un prêt à profil libre, ce sont les contraintes (3.15) et (3.16) qui deviennent :

$$
\begin{aligned}
\operatorname{crd}_{j, k} & =\operatorname{crd}_{j, k-1}-\alpha\left(D_{j, k}, T_{j}\right) \cdot\left(e_{j, k}-T_{j} \cdot \operatorname{crd} d_{j, k-1}-A \cdot m_{j}\right) \\
e_{j, k} & \geq T_{j} \cdot \operatorname{crd} d_{j, k-1}+C_{\min } \cdot d_{j}+A \cdot m_{j} .
\end{aligned}
$$

Malheureusement, les lemmes de dominance (Lem. 3.3 pour les prêts à profil constant et Lem. 3.9 pour les prêts à profil libre) ne tiennent plus lorsqu'une assurance sur CI est associée aux prêts. Prenons l'exemple d'un prêt $P$ de taux $t$ comportant une assurance sur CI de taux $A$. Admettons que ce prêt ait un montant $m$ et une durée $d$. Son coût peut être diminué en décomposant celui-ci en deux prêts $P$ et $P^{\prime}$ de même taux $t$, l'un de durée $d$ et l'autre de durée $d^{\prime}<d$, et dont la somme des montants égale $m$. En effet, le coût des intérêts reste inchangé puisque les deux prêts ont le même taux $t$ alors que le coût de l'assurance devient $d \cdot A \cdot m_{P}+d^{\prime} \cdot A \cdot m_{P^{\prime}}\left(\right.$ avec $\left.m_{P}+m_{P^{\prime}}=m\right)$, ce qui est strictement inférieur à $d \cdot A \cdot m$. En pratique, nous maintenons les coupes de dominance pour des raisons qui apparaîtront dans la section suivante traitant du coût des frais et garanties.

Une assurance peut éventuellement comporter des surprimes lorsque le montant du prêt assuré devient important (supérieur à 500000 euros). Ces surprimes étant rares en pratique (d'autant plus qu'elles disparaissent souvent après négociation), nous n'avons pas jugé utile de les intégrer dans le modèle. En fait, la présence de ces surprimes suggère que le coût d'une assurance soit modélisé comme une fonction affine par morceaux du montant du prêt, entraînant une combinatoire supplémentaire en cas de traitement exact.

\subsection{Garanties et autres frais}

Comme nous l'avons évoqué en introduction, le coût des garanties et autres frais associés à un prêt s'exprime comme une fonction du montant $m$ du prêt, qui viendra s'additionner au besoin total de financement puisque devant être financé par le plan lui-même. Cette fonction, croissante, peut être considérée comme affine par morceaux et continue (bien qu'en pratique, les morceaux de droite ne soient pas toujours parfaitement raccordés). Ainsi, nous noterons cette fonction $G(m)=$ $P_{k} \cdot m+Q_{k}$ pour tout $M_{k-1} \leq m<M_{k}$ (avec $M_{0}=0$ et $M_{K}=+\infty$, où $K$ est le nombre de morceaux). De plus, cette fonction $G(m)$ a des caractéristiques 


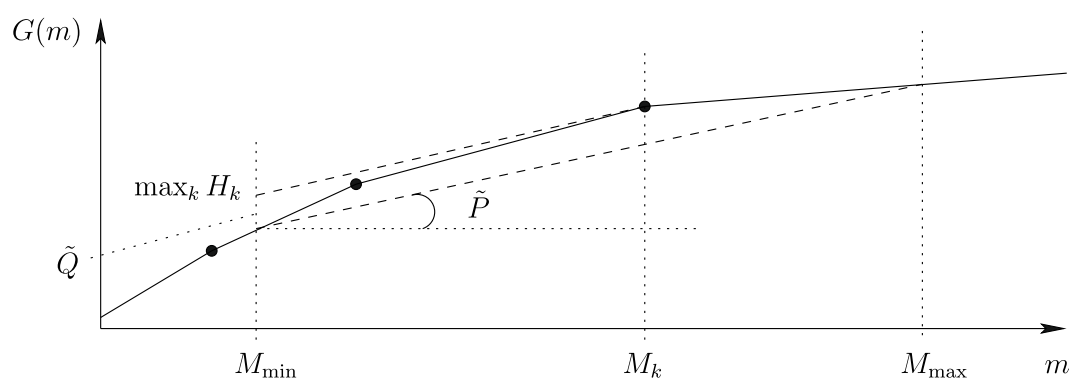

Figure 8. Les paramètres $\tilde{P}$ et $\tilde{Q}$ de l'approximation $\tilde{G}(m)$.

particulières. Le nombre $K$ de morceaux est limité, il ne dépasse pas dix. La pente $P_{k}$ des morceaux de droite décroît à mesure que le montant du prêt augmente $\left(P_{k}>P_{k+1}\right)$, ce qui fait de $G(m)$ une fonction concave. De fait, la fonction satisfait la propriété $G\left(m+m^{\prime}\right) \leq G(m)+G\left(m^{\prime}\right)$ (du point de vue du coût des frais et garanties, contracter deux prêts revient plus cher que de n'en contracter qu'un seul du montant des deux).

Toutefois, intégrer la fonction $G(m)$ de façon exacte dans le modèle linéaire en nombres entiers demanderait l'introduction de variables booléennes, ce qui n'est pas souhaitable dans le contexte temps réel qui nous est imposé. En pratique, les morceaux de $G(m)$ sont tels qu'une fonction affine bien choisie suffit à fournir une approximation acceptable de $G(m)$. Cette fonction, notée $\tilde{G}(m)$, est définie de façon à ce que dans l'intervalle $\left[M_{\min }, M_{\max }\right]$ (avec $M_{\max }$ borné par le besoin $B$ de financement), l'écart $|\tilde{G}(m)-G(m)|$ maximal soit minimum.

Ainsi, la fonction $\tilde{G}(m)$ est construit comme suit. Tout d'abord, le coefficient directeur $\tilde{P}$ de $\tilde{G}(m)$ correspond à la pente entre les points $M_{\min }$ et $M_{\max }$, soit :

$$
\tilde{P}=\frac{G\left(M_{\min }\right)-G\left(M_{\max }\right)}{M_{\min }-M_{\max }} .
$$

Pour chaque point anguleux $\left(M_{k}, G\left(M_{k}\right)\right)$ compris dans l'intervalle $\left[M_{\min }, M_{\max }\right]$, nous calculons sa projection $H_{k}$ en ordonnée sur la droite $m=M_{\min }$ selon la pente $\tilde{P}$ (voir Fig. 8) :

$$
H_{k}=\tilde{P} \cdot\left(M_{\min }-M_{k}\right)+G\left(M_{k}\right) .
$$

L'ordonnée à l'origine $\tilde{Q}$ de la fonction $\tilde{G}(m)$ est alors donnée par :

$$
\tilde{Q}=\frac{\max _{k} H_{k}+G\left(M_{\min }\right)}{2}-\tilde{P} \cdot M_{\min }
$$

De cette façon, la droite $\tilde{G}(m)$ est équidistante des ordonnées $\max _{k} H_{k}$ et $G\left(M_{\min }\right)$ en abscisse $M_{\text {min }}$. La valeur $\max _{k} H_{k}$ peut être déterminée en $O(\log K)$ par dichotomie sur l'ensemble ordonné des points anguleux $\left(M_{k}, G\left(M_{k}\right)\right)$, puisque leurs projections $H_{k}$ induisent une fonction concave. 
En définitive, le coût des frais et garanties est intégré au modèle en remplaçant la contrainte $\sum_{i} m_{i} \geq B$ qui veut que la somme des montants des prêts du plan couvre le besoin de financement par la contrainte :

$$
\sum_{i}\left(1-\tilde{P}_{i}\right) \cdot m_{i} \geq B+\sum_{i} \tilde{Q}_{i} \cdot p_{i}
$$

Il est à noter que les lemmes de dominance (Lem. 3.3 pour les prêts à profil constant et Lem. 3.9 pour les prêts à profil libre) restent vrais en présence de garanties ou de frais, puisque $G\left(m+m^{\prime}\right) \leq G(m)+G\left(m^{\prime}\right)$. Par contre, les Propositions 3.5 et 3.10 caractérisant les plans de financement optimaux ne sont plus exactes. Une remarque intéressante en pratique est que l'impact des assurances sur CI sur la caractérisation du plan optimal (qui tend à fractionner le plan de financement en plusieurs prêts) est contraire à celui des garanties (qui tend à regrouper les prêts); cette observation justifie notre utilisation de la Proposition 3.10 pour modéliser l'amortissement des prêts à profil libre, en dépit de l'adjonction d'assurances, de garanties ou d'autres frais.

\subsection{TAUX EFfectif GLobal et taux D'Usure}

Le taux effectif global (TEG) d'un prêt, dont le mode de calcul est défini dans le Code de la consommation, a été mis en place par l'État afin que les emprunteurs puissent comparer clairement les différentes offres de crédit qui leur sont proposées par les établissements bancaires. En effet, dès lors que des assurances, des garanties et divers frais viennent s'ajouter à la somme des intérêts versés par l'emprunteur, le taux effectif du prêt devient supérieur au taux annoncé (sur lequel le calcul des intérêts est basé). Notons $\bar{m}$ le montant du prêt hors coût des frais et garanties et $\bar{e}_{k}$ l'échéance du palier $k$ toutes primes d'assurances comprises. Le taux effectif du prêt est le taux $t$ qui satisfait l'équation

$$
\bar{m}=\sum_{k=1}^{r} \bar{e}_{k} \cdot \operatorname{VAN}\left(a_{k}, b_{k}, t\right)
$$

où $a_{k}$ (resp. $b_{k}$ ) représente la date de début (resp. de fin) du palier $k$. Toutefois, la définition juridique du TEG n'inclue que les coûts contractuellement obligatoires; certaines assurances sont facultatives (par exemple, les assurances perte d'emploi) et donc ne sont pas intégrés dans le calcul du TEG.

Afin de protéger les emprunteurs de pratiques abusives, le Code monétaire et financier définit un taux au-delà duquel il est illégal de prêter de l'argent, appelé taux d'usure (ou taux usuraire). Ce taux est publié trimestriellement par la Banque de France, chaque type de créance (crédit immobilier, crédit consommation, taux fixe, taux révisable, etc.) ayant un taux d'usure approprié. Ainsi, la loi impose que le TEG d'un prêt ne dépasse son taux d'usure. Ayant noté $U$ le taux d'usure du prêt, il nous suffit d'ajouter la contrainte suivante à notre modèle afin d'assurer la 


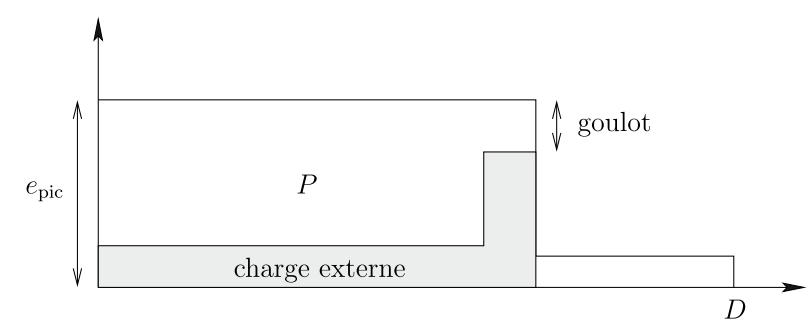

Figure 9. Goulot d'étranglement provoqué par une charge externe.

validité du prêt (la fonction VAN étant monotone décroissante) :

$$
\bar{m} \geq \sum_{k=1}^{r} \bar{e}_{k} \cdot \operatorname{VAN}\left(a_{k}, b_{k}, U\right)
$$

\section{LE PLAN DE FINANCEMENT}

Une fois modélisé toutes les contraintes de prêts, la modélisation des contraintes et objectifs du plan est relativement simple. Soit $e_{j}=\sum_{i} e_{i, j}$ l'échéance totale du plan le mois $j$. Dans le premier mode d'optimisation, c'est-à-dire minimiser le coût du plan de financement du client, la contrainte de capacité s'exprime comme $e_{j} \leq E_{j}$ pour chaque mois $j$, l'objectif étant de minimiser la quantité $\sum_{j} e_{j}$. Dans le second cas, c'est-à-dire lisser l'échéance du plan sur la durée souhaitée par le client, la contrainte de capacité s'exprime comme $e_{j} \leq e_{\mathrm{pic}}$, avec comme objectif minimiser $e_{\text {pic }}$. Un second terme est ajouté à cet objectif afin de minimiser le coût du plan en cas d'égalité sur le premier terme, ce qui peut arriver lorsqu'une charge externe au plan de financement courant induit un goulot d'étranglement dans l'amortissement des prêts (voir Fig. 9).

Comme nous l'avons évoqué dans la section concernant les prêts à profil libre, un terme est ajouté à l'objectif de façon à minimiser le nombre de paliers de l'ensemble des prêts à profil libre présent dans la solution de financement. Enfin, dans les deux modes d'optimisation, la contrainte (5.45) présentée dans la section précédente garantit la couverture du besoin de financement du client. Les grilles de taux étant croissantes, on peut observer qu'il existe toujours un plan optimal couvrant de façon exacte le besoin de financement, dès lors qu'au moins un prêt possède un montant minimum inférieur au besoin de financement (condition qui est vérifiée en pratique).

Notons que la présence d'une durée minimum dans la définition des prêts à profil libre peut conduire a des solutions délicates à défendre d'un point de vue commercial. En effet, admettons qu'un client ait une forte capacité de remboursement lui permettant d'emprunter sur une durée de 3 ans seulement. Si tous les prêts susceptibles de composer le plan de financement ont une durée minimum de 5 ans, alors la durée du plan sera artificiellement allongée jusqu'à 5 ans de façon 


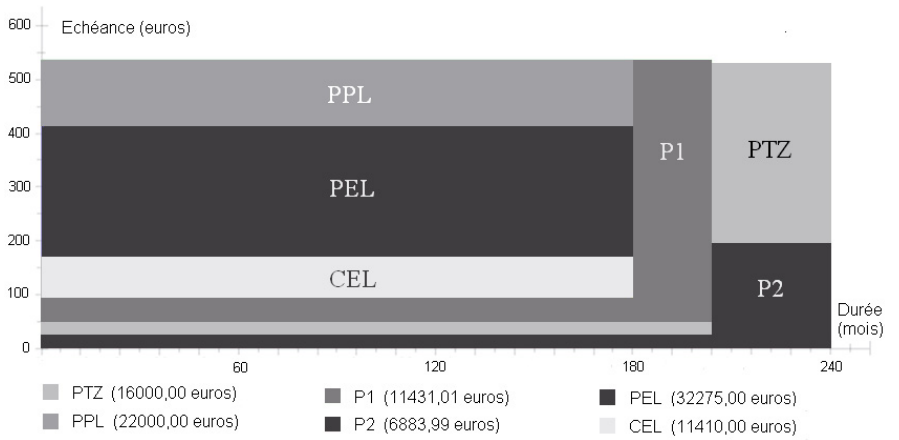

Figure 10. Un plan de financement optimisé à base de 6 prêts : deux prêts du secteur libre (P1, P2), deux prêts épargne logement (PEL, CEL) et deux prêts à taux zéro (PPL, PTZ).

à respecter la contrainte, donnant lieu à une échéance résiduelle de 3 à 5 ans d'un montant légèrement supérieur à $C_{\min }$. Ce type de contrainte n'ayant pas été revue par le service chargée de la définition des produits, nous avons introduit la contrainte $\sum_{j} e_{j} \geq E_{\min }$ avec une constante $E_{\min }$ fixée à quelques dizaines d'euros.

\section{CAS PRATIQUeS}

Pour conclure ce papier, nous présentons quelques exemples de plans de financement calculés par notre module d'optimisation et visualisés à l'aide de notre outil de simulation graphique. La figure 2, présentée en introduction, montre un plan de financement à base de 6 prêts couvrant un besoin de 170000 euros pour une capacité de remboursement de 1000 euros par mois. Dans cet exemple, la durée du PTZ a été raccourcie par l'algorithme comme décrit en Section 4.3.2. La figure 10, présentée ci-dessous, montre un plan de financement également à base de 6 prêts couvrant un besoin de 100000 euros sur une durée souhaitée de 20 ans. Dans ce cas, l'objectif est de minimiser le pic de mensualité, ce qui revient à lisser l'échéance totale du plan sur les 20 ans; la mensualité obtenue est ici de moins de 550 euros. Dans ces deux exemples, nous pouvons observer l'emboitement caractéristique des deux prêts $\mathrm{P} 1$ et $\mathrm{P} 2$ à profil libre, tel que décrit en Section 3.2. Dans les deux cas, la durée du prêt P1 s'arrête à 204 mois (17 ans), qui est une des durées sur lesquelles s'appuie la grille de taux; le taux obtenu sur 17 ans est plus bas que le taux que l'obtient sur 20 ans, taux auquel le montant de P2 est emprunté, ce qui permet de diminuer de façon notable le coût du plan.

La figure 11 montre un plan de financement couvrant un besoin de 100000 euros pour une capacité de remboursement qui décroît progressivement de 700 à 500 euros jusqu'à 10 ans puis croît de 500 à 650 euros. Cet exemple est quelque peu artificiel, mais permet de vérifier la robustesse de l'algorithme face à des données atypiques. L'emprunt principal est réalisé sur un prêt à taux révisable, au taux plus avantageux mais dont la durée a été limitée à 180 mois (15 ans). 


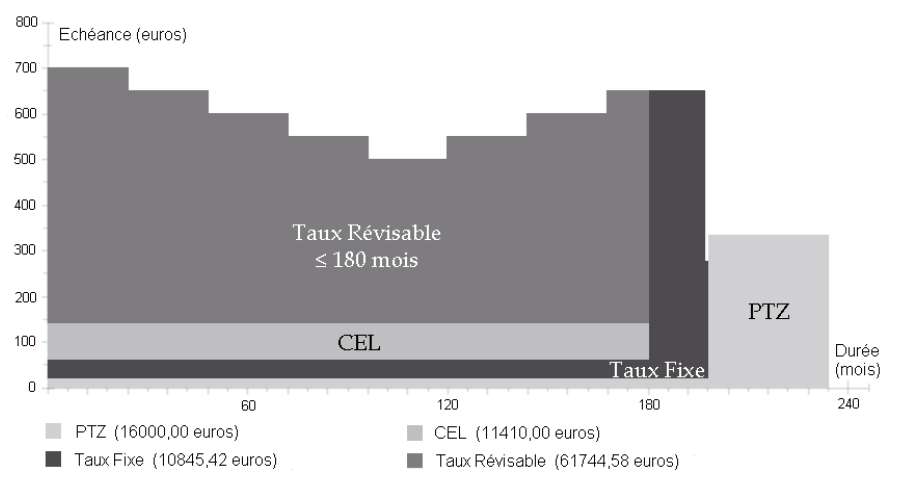

Figure 11. Un plan de financement optimisé à base de 4 prêts : deux prêts du secteur libre (taux fixe, taux révisable), deux prêts réglementés (CEL, PTZ).

\begin{tabular}{|l|c|c|c|}
\hline & Durée (mois) & Coût (euros) & Gain (\%) \\
\hline PF1 & 226 & 58712 & - \\
PF2 & 221 & 55095 & 6.1 \\
PF3 & 217 & 52578 & 10.4 \\
\hline
\end{tabular}

Étudions maintenant quelques cas pratiques. Soit un besoin de financement de 100000 euros et une capacité de remboursement constante de 700 euros par mois. Nous disposons de quatre produits : un prêt à taux fixe, un prêt à taux révisable, un prêt plan épargne logement, un prêt compte épargne logement. À chaque prêt est associé une assurance sur CI et une garantie hypothécaire. La solution de financement basique (PF1) ne contient que le prêt à taux fixe (100000 euros sur 226 mois à $4.75 \%$ ). Une solution plus élaborée (PF2) consiste à intégrer au plan le prêt à taux révisable sur du court terme (23 388.54 euros sur 84 mois à $3.95 \%$ ) pour bénéficier de son taux plus avantageux, tout en limitant les risques liées à une future augmentation des taux (le taux est révisé annuellement, mais ne peut sortir de la fourchette $3.95 \pm 1 \%$ ). Enfin, le meilleur plan de financement (PF3) est composé des quatre prêts : le PEL et le CEL sont intégrés au plan précédent sur 180 mois chacun (voir Fig. 12). Voici un tableau synthétisant la durée et le coût de chacun des plans. La colonne "Gain" du tableau donne le pourcentage d'économie réalisé par le client par rapport à la solution naïve (PF1). Le lecteur notera que le gain obtenu entre le plan naï PF1 et le plan optimisé PF3 est de plus de $10 \%$, soit plus de 6000 euros.

Notre module d'optimisation offre également un retour d'expérience important pour le service du marketing stratégique en charge de la création et de l'évolution des produits. Avant la commercialisation d'un nouveau produit (nouveau type de prêt, nouvelle grille de taux, etc.), le logiciel permet de simuler rapidement des cas typiques tel que rencontrés en agence, et de voir ainsi comment le produit s'intègre aux plans de financement et quellle valeur ajoutée il leur apporte. Reprenons le 


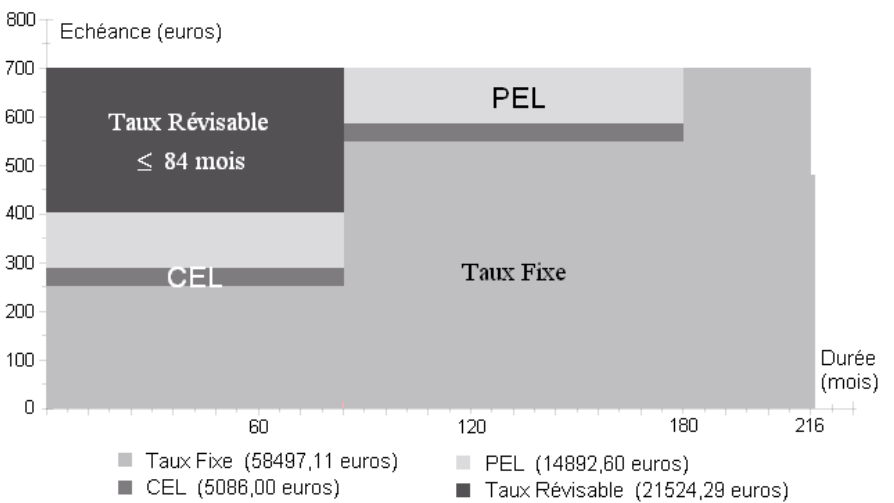

Figure 12. Le plan de financement PF3 à base de 4 prêts : deux prêts du secteur libre (taux fixe, taux révisable), deux prêts réglementés (PEL, CEL).

\begin{tabular}{|c|c|c|c|}
\hline & Durée (mois) & Coût (euros) & Gain (\%) \\
\hline PF1 & 226 & 58712 & - \\
PF2 & 221 & 55095 & 6.1 \\
PF3' & 220 & 54895 & 6.5 \\
\hline
\end{tabular}

cas pratique précédent : un besoin de financement de 100000 euros et une capacité mensuelle de remboursement de 700 euros. Comme nous l'avons montré, le plan PF2 panachant judicieusement taux fixe et taux révisable apporte un gain de $6.1 \%$, en comparaison avec la solution naïve PF1. À présent, construisons le plan optimal PF3' à l'aide de deux prêts à taux fixe ayant les mêmes caractéristiques, en particulier la même grille de taux. Nous obtenons le plan suivant : un premier emprunt de 25850.53 euros au taux de $4.55 \%$ fixe sur 180 mois et un second emprunt de 74149.47 euros au taux de $4.75 \%$ sur 220 mois. Le tableau suivant donne le coût de chacun des 3 plans.

Avec surprise, nous constatons que l'assemblage de deux prêts à taux fixe offre un gain plus important au client que le panachage taux fixe/taux révisable, qui comporte pourtant un risque de hausse du taux révisable. L'outil d'optimisation nous a donc permis de détecter une incohérence dans l'offre commerciale de la banque.

Pour terminer, nous étudions un cas faisant apparaître l'importance de la prise en compte des frais et garanties dans le processus d'optimisation. Soit un besoin de financement de 60000 euros pour une capacité mensuelle de remboursement de 710 euros. Nous disposons de trois produits : un PEL, un CEL et un prêt P à profil libre proposé par la banque. Si l'on optimise sans tenir compte du coût des frais et garanties, alors le plan PF8 obtenu est composé des 3 prêts (voir Fig. 13). La présence du prêt $\mathrm{P}$ au sein de la solution s'explique par le fait que le PEL et le CEL ne peuvent pas saturer la capacité de remboursement (puisqu'à profil constant) 


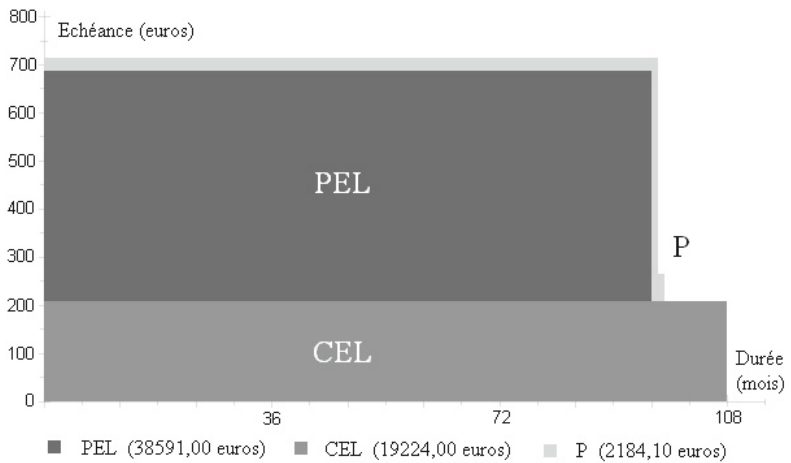

Figure 13. Le plan de financement PF8 à base de 3 prêts : deux prêts réglementés (PEL, CEL) et un prêt du secteur libre $(\mathrm{P})$.

\begin{tabular}{|c|c|c|c|}
\hline & Durée (mois) & Coût hors $G$ (euros) & Coût avec $G$ (euros) \\
\hline PF8 & 108 & 10177 & 13244 \\
PF9 & 120 & 10427 & 12893 \\
\hline
\end{tabular}

et laisse un résidu de capacité exploitable par un prêt à profil libre, même si son taux semble moins avantageux. Si l'on optimise en tenant compte du coût des frais et garanties, alors nous obtenons un plan PF9 composé seulement des prêts PEL (42723 euros sur 96 mois) et CEL (17277 euros sur 120 mois), dont l'échéance totale est légèrement inférieure à la capacité de remboursement. Le tableau cidessous présente les coûts des deux plans, hors coût des frais et garanties (hors $G$ ) et avec coût des frais et garanties (avec $G$ ) :

Omettre le coût des frais et garanties lors de l'optimisation du plan de financement peut donc conduire à des aberrations sur le plan commercial, en l'occurrence une solution de financement plus compliquée et plus chère (tant pour le client que pour la banque).

\section{Conclusion}

Nous avons présenté dans ce papier une approche par programmation linéaire mixte pour traiter un problème d'optimisation de plans de financement immobiliers dans un environnement opérationnel très strict (temps réel, fiabilité numérique, généricité, robustesse). Notre logiciel est aujourd'hui en exploitation dans le réseau d'une grande banque française; un autre établissement majeur, récemment autorisé à entrer sur le marché du crédit immobilier, a depuis intégré celui-ci dans le cadre de la refonte de son système d'information. Étant destiné à l'assemblage complexe de produits de différentes natures, cet outil d'optimisation semble d'autant plus intéressant pour les courtiers en crédits immobiliers.

Cette première expérience dans le monde de la banque de détail laisse entrevoir de nombreux besoins en aide à la décision et optimisation, dus à la complexité 
grandissante des produits commercialisés sur le marché. Les problématiques rencontrées doivent leur difficulté à la grande technicité des métiers de la banque, ainsi qu'aux environnements complexes dans lesquels les algorithmes doivent être intégrés, ce qui leur confère un intérêt certain pour le chercheur opérationnel. Par exemple, un problème que nous n'avons pas abordé ici est l'optimisation de plans de financement immobiliers à destination d'un investissement (et non plus d'une acquisition), qui met en jeu des produits différents (de type prêts in fine) et comporte une forte dimension fiscale.

Un sujet connexe est l'optimisation de plans de financement de projets, et plus particulièrement de grands projets de construction ou de concession tels que l'on peut en rencontrer chez Bouygues Construction (immeubles, ouvrages d'art) ou Colas (routes). À ce propos, nous espérons pouvoir poursuivre les travaux prometteurs [2,3] qui ont été menés sur la simulation et l'optimisation de scénarios pour le financement de Partenariat Public Privé (PPP), contrats nécessitant d'importants investissements généralement financés par emprunts.

\section{RÉFÉRENCES}

[1] G.A. Belyankin, N.N. Korsakov and V.V. Morozov, Optimal loan management. Comput. Math. Model. 12 (2001) 59-64. Traduction de la revue russe Prikladnaya Matematika i Informatika 4 (2000) 77-82.

[2] T. Benoist, Décompositions combinatoires et applications industrielles. Hermès Science Publications, Lavoisier, Paris, France (2007).

[3] T. Benoist and B. Martin, Partenariats Public Privé et recherche opérationnelle. Bulletin de la Société Française de Recherche Opérationnelle et d'Aide à la Décision 18 (2007) 4-7.

[4] P. Bonneau and M. Wiszniak, Mathématiques financières approfondies. 5ème édition, Dunod, Paris, France (1992).

[5] J. Fortunat, C. Fraïssé and J. Varnet, Lexique pratique du financement de l'immobilier. Collection Techniques Bancaires, Revue Banque Édition, Paris (2003).

[6] F. Gardi and A. David, Optimisation de plans de financement immobiliers, in Actes de ROADEF 2006, le 7ème Congrès de la Société Française de Recherche Opérationnelle et d'Aide à la Décision (sous la direction de E.-G. Talbi), Lille, France (2006).

[7] F. Gardi and A. David, Optimisation de plans de financement immobiliers, in Actes des JFPC 2006, les 2èmes Journées Francophones de Programmation par Contraintes (sous la direction de L. Henoque), Nîmes, France (2006) 167-172.

[8] F. Gardi and A. David, Optimisation de plans de financement immobiliers : de la recherche opérationnelle en actuariat bancaire. Bulletin Français d'Actuariat 13 (2007) 107-121.

[9] M.R. Garey and D.S. Johnson, Computer and intractability: a guide to the theory of NPcompleteness. W.H. Freeman \& Co, San Francisco, CA (1979).

[10] M. Girard, Pratique des mathématiques financières. Collection Institut Technique de Banque, Economica, Paris (1992)

[11] A. Makhorin, GLPK : Gnu Linear Programming Kit, version 4.13. (2006), http://www. gnu.org/software/glpk/

[12] Textes officiels du Ministère de l'emploi, de la cohésion sociale et du logement. http://www. journal-officiel.gouv.fr/, http://www.logement.gouv.fr/ 